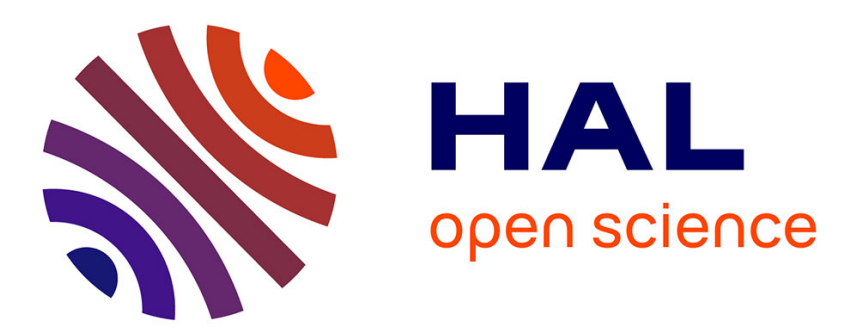

\title{
A Representation of the World Population Dynamics for Integrated Assessments Models
}

Victor Court, Florent Mc Isaac

\section{To cite this version:}

Victor Court, Florent Mc Isaac. A Representation of the World Population Dynamics for Integrated Assessments Models. Environmental Modeling \& Assessment, 2020, 25 (5), pp.611-632. 10.1007/s10666-020-09703-z . hal-02953415

\section{HAL Id: hal-02953415 https://hal-ifp.archives-ouvertes.fr/hal-02953415}

Submitted on 30 Sep 2020

HAL is a multi-disciplinary open access archive for the deposit and dissemination of scientific research documents, whether they are published or not. The documents may come from teaching and research institutions in France or abroad, or from public or private research centers.
L'archive ouverte pluridisciplinaire HAL, est destinée au dépôt et à la diffusion de documents scientifiques de niveau recherche, publiés ou non, émanant des établissements d'enseignement et de recherche français ou étrangers, des laboratoires publics ou privés. 


\title{
A Representation of the World Population Dynamics for Integrated Assessments Models
}

\author{
Victor Court and Florent McIsaac*
}

\begin{abstract}
Using the gross world product (GWP) as the only exogenous input variable, we design a model able to accurately reproduce the global population dynamics over the period 1950-2015. For any future increasing GWP scenarios, our model yields very similar population trajectories. The major implication of this result is that both the United Nations and the Intergovernmental Panel on Climate Change assume future decoupling possibilities between economic development and fertility that have never been witnessed during the last sixty-five years. In case of an abrupt collapse of the economic production, our model responds with higher death rates that are more than offset by increasing birth rates, leading to a relatively larger and younger population. Finally, we add to our model an excess mortality function associated with climate change. Estimates of additional climate-related deaths for 2095-2100 range from 1 million in $\mathrm{a}+2^{\circ} \mathrm{C}$ scenario to 6 million in a $+4^{\circ} \mathrm{C}$ scenario.
\end{abstract}

Keywords: Demographic transition; Global population model; System dynamics; Climate change.

JEL Classification: C62, J11, J21, Q54.

*Victor Court (email: victor.court@ifpen.fr): IFP Energies Nouvelles, IFP School, 1 \& 4 avenue de Bois Préau, 92852 Rueil-Malmaison cedex, France; and Chair Energy \& Prosperity, Institut Louis Bachelier, 28 place de la Bourse, 75002 Paris, France. Florent McIsaac (email: mcisaacf@afd.fr): Agence Française de Développement, 5 rue Roland Barthes, 75012 Paris, France; and Chair Energy \& Prosperity, Institut Louis Bachelier, 28 place de la Bourse, 75002 Paris, France. 


\section{Introduction}

\subsection{Background: the population-economy-climate nexus}

To avoid possible irreversible tipping points highlighted by the Intergovernmental Panel on Climate Change (Stocker et al., 2013), a worldwide agenda has been set in motion to design low-carbon economies and maintain global temperatures below $+2^{\circ} \mathrm{C}$ compared to the pre-industrial era.

To help facing such a challenge, research teams around the globe have built numerous Integrated Assessment Models (IAMs) to try and anticipate the economic and technical challenges associated with the most significant challenge that humanity has ever encountered. In particular, IAMs are used to analyze climate change mitigation scenarios performed by the Working Group III (WGIII) of the IPCC. In the Fifth Assessment Report of WGIII, 31 different IAMs were used to compare 1,184 scenarios (Edenhofer et al., 2014). An important characteristic of this modeling exercise was that the global population always remained exogenous and there was, therefore, no feedback loop between anthropogenic climate change and population. ${ }^{1}$ This is surprising given the recent warning of 15,364 scientists from 184 countries for whom the continuing growth of the global human population is one of the primary driver behind many ecological and societal threats (Ripple et al. (2017, p. 1026), see also Appendix I for a short review of the repeated warnings on overpopulation since the eighteenth century).

To the best of our knowledge, IAMs either assume an exogenous population that corresponds to one of the many projections of the United Nations (UN), or they have an endogenous population module that corresponds to a simplified version of the UN cohortcomponent model which requires three exogenous age-specific time series, namely sex ratios, death rates, and fertility rates. Therefore, IAMs currently take little, if any, consideration of potential feedback between climate change and population dynamics.

\subsection{Goal: designing and testing a world population model for IAMs}

To help fix this issue, the goal of the present article is to design a dynamic world population model that could be added to any kind of IAM. Our model of the world human population is composed of 14 age groups, each of them covering an interval of five years, except for the last group with population aged 65 and over. Each age group is defined by a specific death rate, and the young active population in childbearing age is also define by a fertility rate. The dynamic of these fifteen variables (fourteen group-specific death rates and one fertility rate) needs to be defined to obtain an endogenous age-structured population dynamics. Given our aim to provide a global population model suitable for any kind of IAM, we use the gross world product (GWP) as the only exogenous input variable to determine the birth rate and the fourteen age-specific death rates. In our model, the birth rate and death rates on the one hand, and GWP on the other hand, are related by decreasing sigmoid functions.

We first show that our model accurately reproduces the global population dynamics

\footnotetext{
${ }^{1}$ For a summary of the 31 IAMs used in the Fifth Assessment Report of the Working Group III, see Table A.II.14 in Annex II of the Edenhofer et al.'s (2014, pp. 1309-1310) report.
} 
over the period 1950-2015. Then, to test the prospective outcomes of our model, we use exogenous GWP projections from the first three Shared Socioeconomic Pathways (SSP) developed for the last report from the Working Group III of Edenhofer et al. (2014). The most striking result from our simulation is that given the significant differences between the GWP input of the three SSP scenarios, and contrary to the original population trajectories embedded in these scenarios, there is notable consistency in the population dynamics delivered by our model for any prospective scenario of increasing GWP - either from $S S P 1$, SSP2, or SSP3. Precisely, simulations under the three different exogenous GWP from SSP scenarios are extremely similar and very close to the United Nations medium projection up to 2065, after which date they are clearly above. Given our model, this result makes perfectly sense. Indeed, although we consider different exogenous GWP dynamics in each of the three SSP scenarios, all of them consider GWP levels that are above the 2015 level. Hence, in all our simulations using the GWP from one of the three SSPs, prevailing birth rate and death rates correspond to the lower asymptotic limits of the sigmoid functions we calibrated to define these variables. Our simulation results yield two major implication. First, the SSP scenarios developed for the last Edenhofer et al.'s (2014) report of WGIII take no account of the historical relationship that prevailed between GWP and population from 1950 to 2015. In other words, the population trajectories assumed in SSP scenarios (shown in Figure 4a) are uncorrelated to the GWP trajectories of these same scenarios. Second, the low and even medium prospective variants of the United Nations also assume future decoupling possibilities between economic development and fertility that have never been witnessed during the last sixty-five years.

One major feature of our approach is to suppose a kind of "ergodicity": history has witnessed ever-increasing GWP together with ever-increasing global population (with few local exceptions), therefore, the historically-calibrated functional relationship we define between world population and GWP (eluding the monotonous direction of co-variables) assume that the return to a lower level of GWP implies a turning back to previous birth/death rates. As a consequence of this built-in assumption, we obtain a quite unusual result when a prospective scenario of drastic world economic degrowth is tested in our model: instead of observing a collapse of the future world population as in the World3 model for instance, it increases and gets younger with our modeling framework. To control for this rather counter-intuitive result, we look at the available evidence of economic recession on fertility and death rates. We find that, of course knowledge and technical levels would not vanish instantly in case of economic collapse, but the drastic decrease in GWP per capita that would come with such an event implies that both economic and political institutions and people's behaviors would be heavily disrupted, to the extent that aggregate birth/death rates could indeed return to values characterizing past lower levels of economic development. Furthermore, concerns from the scientific community indicate that future death rates could be significantly altered by climate change (possibly in association with economic degrowth), we also propose a way to implement climate change feedback in our population model.

Therefore, in addition to the fact that the world population model presented in this article could be easily added to any IAM to provide an endogenous population dynamics, it would also help to understand: (i) how climate change is likely to affect population change (through its impact on the death rates, for instance); and (ii) how macroeconomics 
is impacted by such feedback, both in terms of available workforce and aggregate demand.

\subsection{Organization of the rest of the article}

The remaining of this paper is organized as follows. In Section 2, we review the literature on world population models in order to understand why these models are rarely used in larger economic models such as IAMs. In Section 3, we present empirical facts regarding the birth rate and age-specific death rates in relation to gross world product (GWP). Those relations justify the set of equations defining our model of the world human population. The first part of Section 4 is dedicated to testing the ability of the model to reproduce the historical global population estimates of the United Nations. In a second part, prospective simulations of the model are performed in order to assess its sensitivity under three exogenous ever-increasing GWP scenarios. In Section 5, we discuss the need for changes to the model under a drastic regime change such as an economic collapse, or a feedback from climate change. Finally, a summary of the contributions to this article is given in Section 6, along with recommendations for future research.

\section{Review of global population models}

As summarised in Table 1, when we reviewed the literature on global human population models, we identified four different classes of analytical frameworks that we labeled 'overlapping generations models' (2.1), 'carrying capacity models' (2.2), 'World3-like models' (2.3), and 'UN cohort-component models' (2.4).

\subsection{Overlapping generations models}

The overlapping generations model (OLG) is one of the dominant neoclassical analytical frameworks for studying macroeconomic dynamics, but in contrast to the Ramsey-CassKoopmans neoclassical growth model in which individuals live infinitely, in the OLG model individuals live for a limited time, long enough to overlap with at least one period of another agent's life (de La Croix and Michel, 2002). Accordingly, in an OLG model, population is represented by two (children and adult) or three (children, young adult, and old adult) representative generations at each given time step. Usually, at each time step, the representative young adult allocates its unitary time endowment between child rearing and effective labor force participation to earn a wage. The wage income is either spent through direct consumption, invested in children's education, or saved to supply the capital investment market if it is modelled. The advantage of this framework lies in its simplicity and tractability. The problem is that this framework requires several strong hypotheses, namely that the representative household is unisex, and that the young adult maximize a utility function (that needs to be defined) under a time-budget constraint. In terms of resolution, this kind of model also needs the input of two variables at each time step, namely an equilibrium wage and the average level of education per capita or human capital per capita. These different constraints imply that an OLG might not be the best modeling choice for IAMs. 


\subsection{Carrying capacity models}

Since von Foerster et al. (1960), several articles have developed simple models where, omitting any consideration of age or sex structures, the world population is represented by a single variable that follows a (set of) differential equation(s). ${ }^{2}$ The dynamics of such models depend on the exogenous definition of several parameters, with Earth's carrying capacity being the most crucial parameter. The term carrying capacity is generally used in the literature to frame a stationary world population size that would ensure the stable coexistence of the biosphere and civilization in the long term. The review of carrying capacity estimates performed by Cohen (1995) shows that (up to 1995) the 65 lower (respectively upper) bounds on human population was 7.7 (respec. 12) billion, with extreme estimates ranging from 1 to 1000 billion. In addition to the difficulty of estimating the carrying capacity parameter, which should furthermore evolve with environmental change, some of those models, such as Dolgonosov (2016) and Okuducu and Aral (2017), introduce another endogenous variable representing the informational, or knowledge, state of humanity. The general knowledge-related measure of civilization's progress, and the additional parameters that come with its formulation, hardly find a correspondence with real world data, which may cast further doubts on the calibration and prospective results of those models. ${ }^{3}$ Alternatively, in a method closer to standard economic theory, Cai (2012), Guerrini (2013), and others introduce capital accumulation and production decisions to capture humanity's development level rather than a knowledge-related variable. Nevertheless, it is clear that the different 'carrying capacity models' can hardly be introduced into IAMs, because their representation of the global human population is too simple (no age structure), and abstract (relying on the carrying capacity of the Earth and other endogenous parameters).

\subsection{World3-like models}

A third class of models, hereafter labeled 'World3-like models', corresponds to more complex analytical frameworks based on system dynamics. Such models explicitly feature an age structure by defining births and death rates that are specific to each age group. As a result, such models do not require the uncertain estimation of a parameter representing the carrying capacity of the Earth. Because of their higher degree of realism, models of this second group seem more meaningful for IAMs, yet they have not been widely implemented. This is because these models derive from the World3 model of Meadows et al. (1972), and as this original source, one of their drawbacks is their lack of clarity and transparency. In particular, it is difficult to understand what exactly drives the dynamics of such kind of models. In recent versions, articles that adopt such frameworks either summarize a partial set of equations with a graphical representation (Cole and Flenley, 2008; Eberlein and Thompson, 2013; Navarro et al., 2017); or, on the contrary, suffer from high dimensionality and complexity (Micó et al., 2008, 2006; Sanz et al., 2014).

\footnotetext{
${ }^{2}$ Akaev and Sadovnichii (2010) is a good introduction to this class of models.

${ }^{3}$ Aral (2014) has introduced climate change into an information-based model of this kind.
} 


\subsection{UN cohort-component models}

Finally, a fourth class of global population models, hereafter labeled 'UN cohort-component models', uses the United Nations' (2017a) accounting framework for fertility, mortality, and migrations. In such models, population is divided between males and females and five-year age groups. Inflows and outflows of each five-years age groups depends on three age-specific variables, namely sex ratios, death rates, and fertility rates. This model is undoubtedly precise, but such a precision comes at the expense of a major drawback: sex ratios, death rates, and fertility rates must be defined exogenously for each age group. In UN's studies, econometric analyses are used to compute these variables and used them as input to compute world and regional populations estimates. Because of these methodological requirements, it is not surprising that this population representation is not implemented in IAMs to generate an endogenous population. Rather, population estimates of the UN are used as exogenous inputs in IAMs, but consequently there is no possible interaction between population and crucial variables of IAMs such as GDP, technical levels, or climate-related indicators.

Table 1: Advantages and flaws of different world human population models.

\begin{tabular}{lll}
\hline & Advantages & Flaws \\
\hline Overlapping generations models & $\begin{array}{l}\text { Tractable problem with } \\
\text { closed-form solutions }\end{array}$ & $\begin{array}{l}\text { Assumes utility function, } \\
\text { need equilibrium wage }\end{array}$ \\
Carrying capacity models & $\begin{array}{l}\text { Tractable problem with } \\
\text { closed-form solutions }\end{array}$ & $\begin{array}{l}\text { Need to define the carrying } \\
\text { capacity of the Earth }\end{array}$ \\
World3-like models & $\begin{array}{l}\text { Realist 5-years } \\
\text { age structure }\end{array}$ & $\begin{array}{l}\text { Opacity regarding } \\
\text { driving mechanisms }\end{array}$ \\
UN cohort-component models & $\begin{array}{l}\text { Realist sex and } \\
\text { 5-years age structure }\end{array}$ & $\begin{array}{l}\text { Need for three age-specific } \\
\text { exogenous time series }\end{array}$ \\
Present article & $\begin{array}{l}\text { Realist 5-years age structure, } \\
\text { GWP is the only exogenous driver, } \\
\text { model remains comprehensible }\end{array}$ & $\begin{array}{l}\text { Possible difficulty to } \\
\text { obtain closed-form } \\
\text { solutions }\end{array}$ \\
\hline
\end{tabular}

We argue that there is a lack of intermediate-size human population models that would both avoid the flaws and combine the advantages that respectively affect the four classes of models described supra and in Table 1. If possible, such an intermediate model should offer closed-form solutions without requiring the estimation of abstract parameters, in particular the carrying capacity of the Earth. In the meantime, such a model should be complex enough to feature an age structure without presenting the flaws of a highly dimensional black box. 


\section{Methods: from the data to the model}

Prior to introducing the dataset, let us first sketch the methodology that will motivate the choices made throughout this section. The global population, $N$, is divided into $n$ age groups, $N_{i}$, so that

$$
N=\sum_{i=1}^{n} N_{i}
$$

The dynamics of each group $i$ follows the law of motion,

$$
\dot{N}_{i}=I_{i}-O_{i}, \quad \forall i \in\{1, \ldots, n\}
$$

where, $I_{i}$, is the inflow of population within the age group $i$ (births or changes in age group), and, $O_{i}$, is the outflow of population within the age group $i$ (deaths or changes age group). After introducing the dataset used to calibrate our model (3.1), this section will focus on the determination of parametric forms for the birth rate and death rates (3.2) that are necessary to define variables $\left(I_{i}, O_{i}\right)$ for each age group.

\subsection{Ensuring the stock-flow consistency of the dataset}

Figure 1a plots the stock of population from 1950 to 2015 for 14 age groups $\left(N_{1}, \ldots, N_{14}\right)$ at a five-year frequency retrieved from the United Nations (2017b). ${ }^{4}$ The $i^{\text {th }}$ group, $N_{i}$, for $i \in\{1, \ldots, 13\}$, represents the $[5(i-1), 4+5(i-1)]$ years-old age group. The last group, $N_{14}$, represents the population over 65 years-old. As a result of data granularity, the unit time between two periods ( $t$ and $t+1$ ) corresponds to 5 , which is the number of years during which individuals remain in a given age-group.

When using the data for economic modeling purposes, a minimum of four distinct cohorts $\left(C_{1}, \ldots, C_{4}\right)$ can be identified out of the 14 age groups:

- $C_{1}:=\sum_{i=1}^{3} N_{i}$, the young inactive population from age 0 to 14 ;

- $C_{2}:=\sum_{i=4}^{10} N_{i}$, the young active population in childbearing age from 15 to $49 ;{ }^{5}$

- $C_{3}:=\sum_{i=11}^{13} N_{i}$, the old active population from 50 to 64 ; and

- $C_{4}:=N_{14}$, the retired population away from the job market from 65 and over. ${ }^{6}$

${ }^{4}$ More precisely, two time series are used in this section: (i) File POP/7-1: Total population (both sexes combined) by five-year age group, region, sub-region and country, 1950-2100 (thousands); and (ii) File MORT/4-1: Deaths (both sexes combined) by five-year age group, region, sub-region and country, 1950-2100 (thousands).

${ }^{5}$ As a consequence of this modeling choice, we ignore the fact that some individuals, especially men, can have children after 49.

${ }^{6}$ Of course, in many countries there are now indications to push the retirement age further than 65 . But here we are constrained by the United Nations data, which only has one age group above 65. 
Therefore, $C_{2}+C_{3}$ represents the active population and would define the denominator of the employment rate in a broader economic model. The choice of 14 different age groups is motivated by the fact that transfer of population between one (economically meaningful) cohort to another strongly depends on the population structure itself. Indeed, if one assumes a constant (average) share of population transfer between cohorts (as in 'World-3 type models'), then a rather strong bias would appear in situation like the baby boom at the aftermath of WWII. The consequences of a misrepresentation of the first three cohorts can lead to misleading measurements of the (past and future) workforce and population inflow. However, we chose a simpler aggregated characterization for the last cohort dynamics as it is not the primary focus of our paper. Moreover, throughout our model we assume (i) a strict gender parity in population, and (ii) a uniform age distribution within each of the fourteen 5-year age groups.

To ensure the stock-flow consistency of the data, we first define the global inflow of the population system, i.e., $I_{1}$. Let $N^{d}=\sum_{i=1}^{14} N_{i}^{d}$ be the total number of deaths over all groups, with $N_{i}^{d}$ the number of deaths within group $i$. Intuitively, the global number of births, $I_{1}$, is

$$
I_{1}:=\dot{N}+N^{d} .
$$

Once the number of births is computed, it is easy to obtain the population transfer from group 1 into group $2, T_{1,2}$, as

$$
T_{1,2}:=I_{1}-\dot{N}_{1}-N_{1}^{d} .
$$

Therefore, we identify Eq. 1 for the dynamics of the age group $N_{1}$ to be

$$
\dot{N}_{1}=I_{1}-\underbrace{O_{1}}_{=T_{1,2}+N_{1}^{d}} .
$$

The logic is similar for groups $N_{2}, \ldots, N_{13}$ when identifying $I_{i}:=T_{i-1, i}$, and $O_{i}:=T_{i, i+1}+$ $N_{i}^{d}$. As expected, the last cohort's outflow is only characterized by $O_{14}:=N_{14}^{d}$. Therefore, we are able to determine each variable of Eq. 1 for the fourteen age groups, and consequently we can compute the global population $N .^{7}$

\subsection{Determining parametric forms for the birth and death rates}

Inflows and outflows in our model are defined by 15 dynamic variables: (i) the birth rate determining the inflow in the first age group, $I_{1}$, (ii) the fourteen specific death rates of any $i^{\text {th }}$ age group allowing a distinction between aging and death outflows. According to the literature, the birth rate depends on various determinants, such as

- the education level (Becker et al., 2010; Murphy, 2015),

- the demand for human capital (Galor and Mountford, 2008; Greenwood and Seshadri, 2002),

\footnotetext{
${ }^{7}$ Logically, the transfered population of a given group, $T_{i, i+1}$, should be strictly equal to the population number of the next group after taking into account the number of deaths, $N_{i+1}-N_{i+1}^{d}$. However, we observed in the data a mismatch between those variables. We argue that this discrepancy comes from the fact that death numbers in each 5-year age groups are estimated every five years by the UN from mid-year to mid-year.
} 
- the extent of secularization (Peri-Rotem, 2016),

- marriage patterns (Carmichael et al., 2016),

- women empowerment (Diebolt and Perrin, 2013; Murphy, 2015),and

- wealth inequalities (Cummins, 2013).

Similarly, death rates vary according to many different variables, such as

- the level and quality of nutrition (De Onis, 2000),

- the prevalence of contagious and infectious diseases depending personal hygiene and public health measures (Luby, 2017),

- the significance of chronic and degenerative diseases (Lee, 2003, p. 171),

- the severity of air pollution (Di et al., 2017), and

- the frequency of armed conflict (Guha-Sapir and van Panhuis, 2003).

Since the aim of this paper is to provide a simple world population model that could be included into any IAM, the interactions between the different factors listed supra, and the analysis of their causal impacts on population growth are out of the scope of this paper. Instead, we chose the per capita gross world product (with GWP retrieved in trillion constant 2010 US\$ from the World Bank (2018)) as the exclusive correlative - and not explanatory variable for the birth rate and for the evolution of age-specific death rates. Notwithstanding the well-known drawbacks surrounding GWP (hereafter $Y$ ) as an indicator of wealth (or prosperity), we argue below that this indicator remains a good proxy for the explanatory factors listed supra in determining the evolution of the birth and death rates. ${ }^{8}$ Thus, the approach proposed here is phenomenological - rather than micro-funded - because it relies on relations that emerged from data observed at the global long-term scale.

\subsubsection{Global birth rate as a function of GWP per capita}

Figure 1b shows the scatter plot of the global birth rate and GWP per capita. It displays a $S$-shaped relationship between the two variables. Therefore, we model the evolution of the global birth rate, $B R:=I_{1} / C_{2}$, as a sigmoid function of per capita GWP, $Y / N$.

$$
B R:=\overline{B R}+\frac{\underline{B R}-\overline{B R}}{(1+\exp (-\delta(Y / N-\phi)))^{\frac{1}{\nu}}}
$$

where $\overline{B R}$ and $\underline{B R}$ respectively represent the upper and lower asymptotes, $\delta$ is a slope parameter, $\phi$ is the GWP/capita value determining the inflection point, and $\nu$ is a parameter that controls the curvature of the function near the asymptotes.

\footnotetext{
${ }^{8}$ Regarding birth, our approach is in line with Becker (1960) who suggests that the rise in income induces a fertility decline, because the positive income effect on fertility is dominated by a negative substitution effect induced by the rising opportunity cost of raising children.
} 
Figure $1 \mathrm{~b}$ also displays the result obtained from minimizing the sum of squared errors between the historical data (black dots) and the model of Eq. 2 (blue line). ${ }^{9}$ As can be seen, the high asymptote of 0.39 births per young active individual (i.e, 15-49 years old) every five years is about twice as high as the low asymptotic of 0.18 births per young active individual every five years.

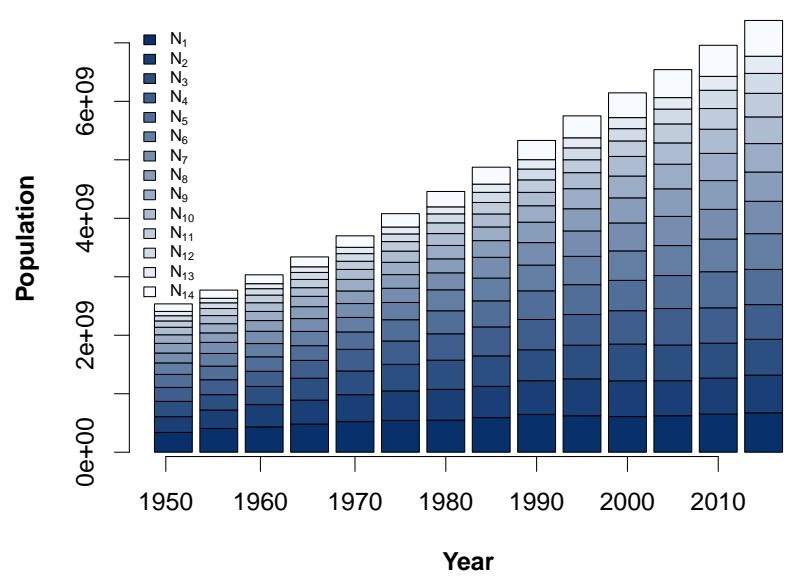

(a)

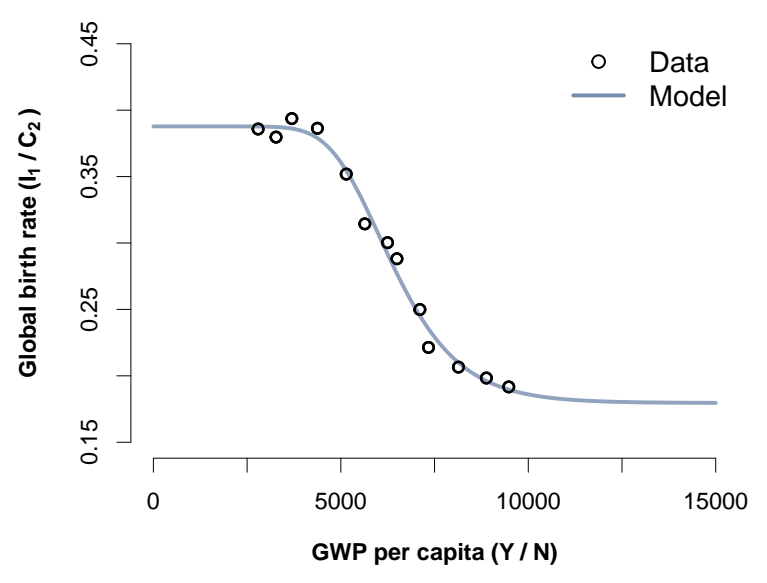

(b)

Fig. 1. (a) world population structure by five-year age groups, 1950-2015; and (b) global birth rate as a function of per capita GWP. Data source: Kremer (1993) from 1700 to 1950, United Nations's (2017b) medium estimate from 1950 to 2100; World Bank (2018).

\subsubsection{Age-specific global death rates as functions of per capita GWP}

We also observed a robust decreasing relationship between the global death rate of every age group and the GWP per capita. However, depending on the age group, the functional relationship can be either exponential or sigmoid. We choose to model the evolution of the different global death rates, $D R_{i}:=N_{i}^{d} / N_{i}$, as decreasing sigmoid functions of GWP per capita, $Y / N$, for two reasons. First, the sigmoid function is a more general formulation of the exponential. This means that in the presence of death rate estimates for very low levels of GWP/capita (that we lack for the moment), one would have more chances to obtain a good fit of the function with respect to the data, no matter what the form of the scatter plot. But mostly, an exponential relationship would imply that death rates tend towards infinity as per capita GWP tends towards zero. Such an assumption would not make sense because an individual surviving alone on an island would certainly have a non-infinite death rate even though the economic system he is living in would match the description of a 'zero GDP' economy. More prosaically, data at the country and world levels shows that prior to demographic transition (i.e., when economic development and growth are low), death

\footnotetext{
${ }^{9}$ The minimization procedure consists in findings the parameters that minimize the sum of squared residuals. In practice, we selected the model that minimizes a BFGS algorithm employed with various initial points. More details are available upon request. Best-fit values of parameters are reported in Tables II.1 and II.2 of Appendix II.
} 
rates are high but globally stable relatively to modern standards (Lee, 2003, pp. 170-173). Hence, at low levels of economic development, assuming an upward asymptote for death rates makes more sense than an exponential negative relationship.

$$
D R_{i}=\overline{D R_{i}}+\frac{\frac{D R_{i}}{-D R_{i}}}{\left(1+\exp \left(-\delta_{i}\left(Y / N-\phi_{i}\right)\right)\right)^{\frac{1}{\nu_{i}}}}
$$

where, for any age group $i, \overline{D R_{i}}$ and $D R_{i}$ respectively represent the upper and lower asymptotes, $\delta_{i}$ is a slope parameter, $\phi_{i}$ is the GWP/capita value determining the inflection point, and $\nu_{i}$ is a parameter controlling for/that controls the curvature of the function near the asymptotes.

Figures $2 \mathrm{a}, 2 \mathrm{~b}, 2 \mathrm{c}$, and $2 \mathrm{~d}$ show the results of the minimization procedure (defined supra) for Eq. 3. In terms of orders of magnitude, the $N_{1}$ group of 0-4 years-old is very distinguishable since its upper asymptote is twice as large as those of groups $N_{2}$ to $N_{13}$. The $N_{14}$ group of over 65 years-old is also discernible as part of the fragile population.

The following section is dedicated to the results obtained with our model. We first present our whole model using matrices and analyze its equilibrium properties (4.1). Then, we perform various simulation runs: an assessment of the historical performance of our model (4.2); and an appraisal of the model's prospective sensitivity under four different exogenous GWP trajectories (4.3).

\section{Results: testing the model in the past and future}

\subsection{Full theoretical model and its properties}

We now turn to the presentation of our theoretical model and the analysis of its equilibrium properties. Recall that the unit time between consecutive periods ( $t$ and $t+1)$ corresponds to the number of years during which individuals remain in a given age-group (i.e., 5 years in the UN data presented in the previous section). Therefore, the outflow of population among age groups $i \in\{1, \ldots, 13\}$ will be the entire population in each group. For each of these outflows, the population that survived remains in the system. This share of population for a given age-group $i$ is represented by $D R_{i}^{*}:=1-D R_{i}$. Let $\vec{N}$ be the column vector of all the age groups. The theoretical model is linear and can be written as

$$
\dot{\vec{N}}=A \vec{N}
$$

where the $A$ matrix is explicitly provided in System 4. 


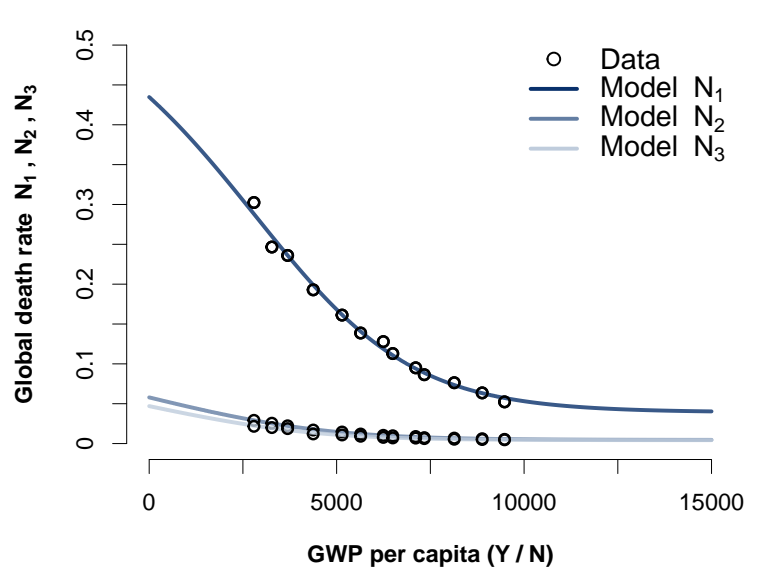

(a) Cohort $C_{1}$

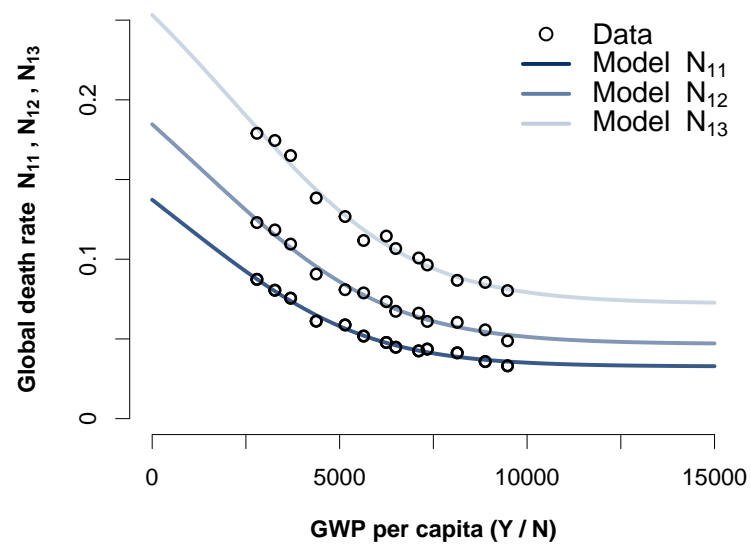

(c) Cohort $C_{3}$

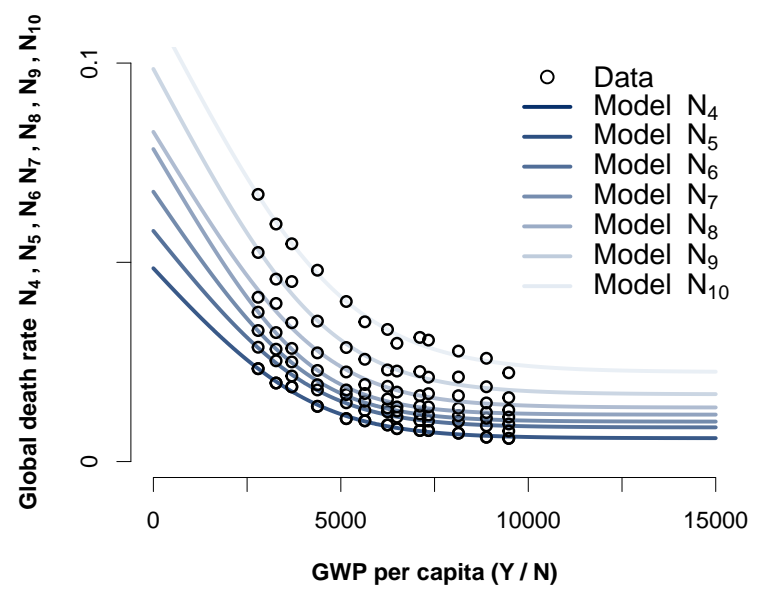

(b) Cohort $C_{2}$

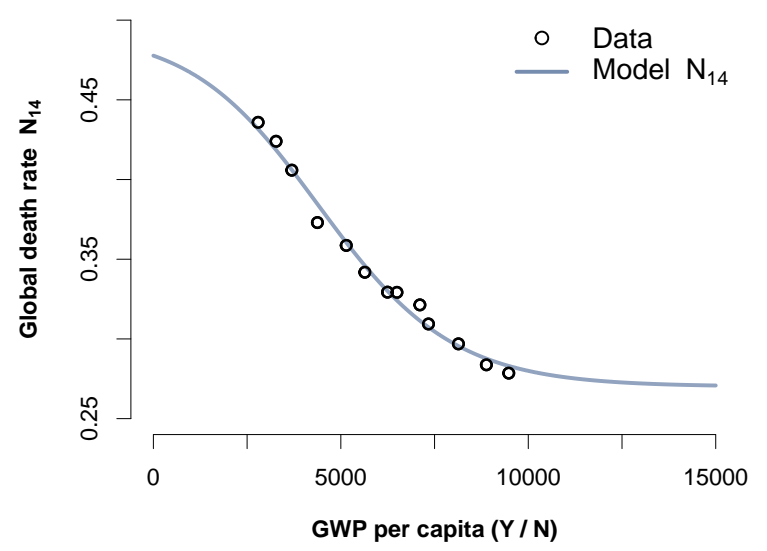

(d) Cohort $C_{4}$

Fig. 2. Global death rates for cohort $C_{1}$ (a), $C_{2}$ (b), $C_{3}$ (c), and $C_{4}$ (d) as functions of per capita GWP. Data source: United Nations's (2017b) medium estimate from 1950 to 2100; World Bank (2018). 


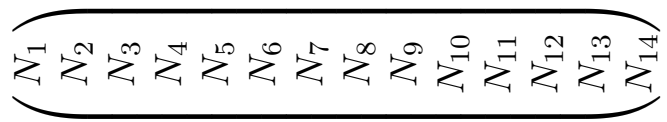

$$
\begin{aligned}
& 0000000000000 \frac{\overbrace{}^{4}}{4} \\
& 0000000000007 \frac{n^{n}}{2} \\
& 000000000007 \frac{{ }^{*}}{2} 0 \\
& \text { 0 0 0 0 o o o o o } \\
& \frac{1}{\pi} 00000000 \frac{7}{4} 000
\end{aligned}
$$

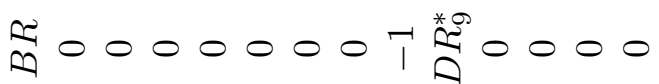

$$
\begin{aligned}
& \left.\begin{array}{lllllllllllllll}
\pi & 0 & 0 & 0 & 0 & 0 & 0 & \frac{a}{\pi} & 0 & 0 & 0 & 0 & 0
\end{array}\right\}
\end{aligned}
$$

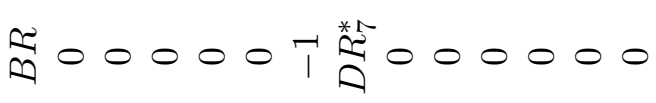

$$
\begin{aligned}
& \text { 公 } 0000 \text { H }
\end{aligned}
$$

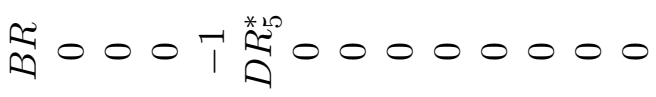

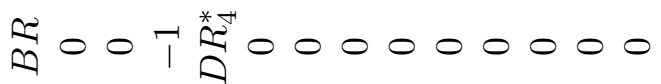

$$
\begin{aligned}
& 00 \text { Ү } \\
& 0 \text { H }
\end{aligned}
$$

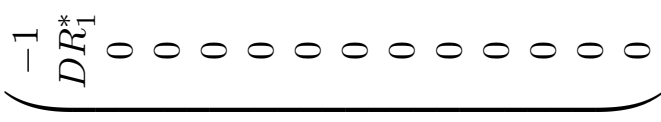

$$
\begin{aligned}
& \text { II }
\end{aligned}
$$

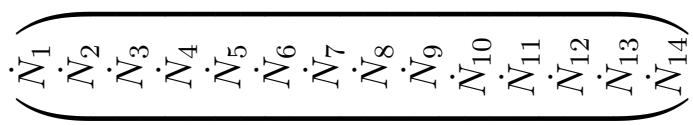


Turning to the equilibrium properties of System 4, we focus on the analysis of the $A$ matrix. As the dimensionality of matrix $A$ is relatively high, let us first consider a lower dimensional system that may be more easily understood. Using $M$ instead of $N$, we consider the following three-dimensional system

$$
\left(\begin{array}{l}
\dot{M}_{1} \\
\dot{M}_{2} \\
\dot{M}_{3}
\end{array}\right)=\underbrace{\left(\begin{array}{ccc}
-1 & B R & 0 \\
1-D R_{1} & -1 & 0 \\
0 & \left(1-D R_{2}\right) & -D R_{3}
\end{array}\right)}_{=: B}\left(\begin{array}{l}
M_{1} \\
M_{2} \\
M_{3}
\end{array}\right)
$$

System 5 has one obvious equilibrium point, $(0,0,0)$. Its local asymptotic stability will depend on whether the linear matrix, $B$, is a negative-definite matrix. The second obvious equilibrium is $(+\infty,+\infty,+\infty)$ retrieved from the change of variable $\left(1 / M_{1}, 1 / M_{2}, 1 / M_{3}\right)$. Its local asymptotic stability will depend on whether the linear matrix, $-B$, is a negativedefinite matrix. The third (set of) equilibria comes under very specific conditions: (i) (1$\left.D R_{1}\right) B R=1$; and (ii) $M_{3}=\left(1-D R_{2}\right) /\left(D R_{3}\right) M_{2}=\left(1-D R_{2}\right) /\left(B R \times D R_{3}\right) M_{1}$. Condition (i) implies that the determinant of the matrix $B$, $\operatorname{det}(B)=-D R_{3}\left(1-B R\left(1-D R_{1}\right)\right)$ equals zero, which would make this set of equilibria structurally unstable. Therefore, the system is asymptotically unstable in the sense that there is no stable equilibrium besides zero or infinity, under specific specifications.

Interestingly, when computing the determinant of the full system, we find a result that is akin to the determinant of the $B$ matrix,

$$
\operatorname{det}(A)=D R_{14}\left[1-B R \sum_{i=3}^{9} \prod_{j=1}^{i} D R_{j}^{*}\right] .
$$

As $B R<1$ and $0<D R_{i} *<1 \forall i, \operatorname{det}(A) \neq 0^{10}$ and, therefore, no finite positive equilibrium can be found. ${ }^{11}$ As a consequence, we acknowledge that the model we designed in the present article would not be suitable for the debate on the global carrying capacity of planet Earth.

Moreover, System 4 allows us to derive the exact solution and to estimate the propagation errors. The solution of the system is

$$
\vec{N}_{t}=e^{A(Y)\left[t-t_{0}\right]} \vec{N}_{t_{0}}
$$

where $\vec{N}_{t_{0}}$ is the initial condition, and $e^{A(Y)}:=\sum_{k \in \mathbb{N}} A(Y)^{k} / k$ !. If one assumes that the initial condition may suffer from measurement errors, the exact solution allows us to assess its impact on the results. For instance, let's assume that the correct measurement of the population is $\tilde{\vec{N}}_{t_{0}}=\vec{N}_{t_{0}}+\varepsilon$, with $\varepsilon \in \mathbb{R}^{14}$. Therefore, the propagation of the errors are as

\footnotetext{
${ }^{10}$ Except for a very particular combination of variables that we do not have in our analysis and calibration.

${ }^{11}$ Moreover, we note that under the suggested calibration, numerical simulations of the $A$ matrix show that only one eigenvalue remains positive for all levels of GWP per capita. This makes System 4 asymptotically unstable.
} 
follows

$$
\begin{aligned}
\tilde{\vec{N}}_{t}-\vec{N}_{t} & =e^{A(Y)\left[t-t_{0}\right]} \tilde{\vec{N}}_{t_{0}}-e^{A(Y)\left[t-t_{0}\right]} \vec{N}_{t_{0}}, \\
& =e^{A(Y)\left[t-t_{0}\right]}\left(\overrightarrow{\vec{N}}_{t_{0}}-\vec{N}_{t_{0}}\right), \\
& =e^{A(Y)\left[t-t_{0}\right]} \varepsilon .
\end{aligned}
$$

As expected, the propagation of the error will increase exponentially over time at a speed that is dictated by the $A$-matrix. ${ }^{12}$ We note that despite the nonlinearity of the model, the propagation errors are linear with respect to $\varepsilon$.

Despite some imperfections, we believe that our model is a useful tool for short- and even medium-term simulation purposes. This is illustrated by the rest of this section. We first assess the ability of our model to reproduce past population estimates (4.2). Then, we test the sensitivity of our model to three exogenous ever-increasing GWP scenarios (4.3). For all simulations, the unit of time-between $t$ and $t+1$-is 5 years.

\subsection{Fit of the model to the historical data}

To assess the empirical robustness of our model, we present the results from its back-testing analysis over the last century. Using values from Table II.1 and Table II.2 in Appendix II, we ran our world population model from 1950 onward with the historical GWP per capita already used in Section 3. We did not perform a historical fit further back because of the lack of reliable estimates of GWP/capita prior 1950 (for example some available years are 1940, 1913, 1870, and 1820 from Bolt et al. (2013)). It is worth noting that the rare GWP/capita estimates prior 1950 are the subject of continuing debates (e.g., Jerven (2012) and Prados de la Escosura (2016)). Moreover, to comply with our framework, it would be necessary to carry out precarious interpolations to obtain GWP/capita estimates with a 5year interval. For these two reasons, we restrict the model estimation to the post-WWII period.

Figure 3a shows that the back-testing simulation is excellent for the first ten periods of time (1950-2000), after which the model starts to slightly underestimate the actual historical population. The initial underestimation in 2000 worsened up until 2015 due, most probably, to accumulated errors. A comparison of the real data age structure in 2015 with the model output made in Figure 3b shows that the overall underestimation of the model is due to a 0.06 billion overestimation for group $N_{14}$ (65+ years-old) that is more than compensated by a 0.05 billion underestimation for group $N_{6}$ (25-29), a 0.04 billion underestimation for group $N_{10}$ (45-49), a 0.03 billion underestimation for group $N_{9}$ (4044), a 0.02 billion underestimation for groups $N_{2}$ (5-9), $N_{5}$ (20-24), $N_{7}$ (30-34), and $N_{11}$ (50-54), and to a lesser extent, a 0.01 billion underestimation for groups $N_{1}(0-4)$ and $N_{3}$ (10-14).

\footnotetext{
${ }^{12} \mathrm{An}$ quantitative assessment of the propagation errors of the calibrated model is provided in Appendix III.
} 


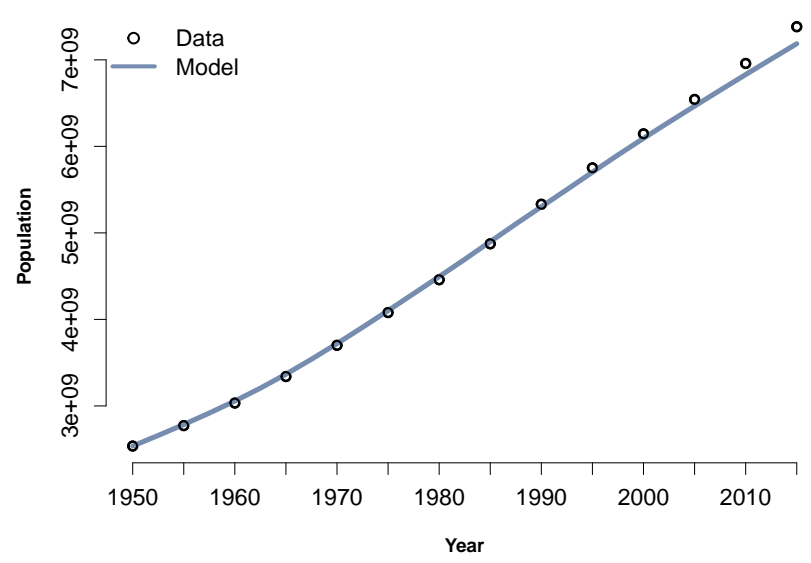

(a) Population level, 1950-2015.

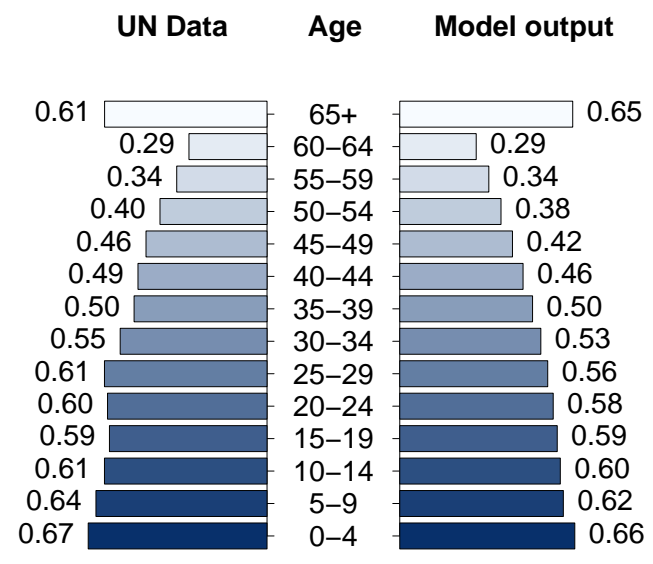

(b) Population pyramids in 2015 (in billions).

Fig. 3. UN historical data vs. model output.

\subsection{Prospective simulations under ever-increasing exogenous GWP sce- narios}

\subsubsection{Choice of exogenous GWP scenarios}

To analyze the sensitivity of our model to the GWP per capita input, we use the first three Shared Socioeconomic Pathways (SSP) developed for the last report from the Working Group III of Edenhofer et al. (2014):

- SSP1 - Taking the Green Road (Low challenges to mitigation and adaptation): The world shifts gradually, but pervasively, towards a more sustainable path, emphasizing more inclusive development that respects perceived environmental boundaries, triggering a large scale learning effect and positive externalities that boost growth relative to the business-as-usual (i.e., SSP2) scenario.

- SSP2 - Middle of the Road (Medium challenges to mitigation and adaptation): The world follows a path in which social, economic, and technological trends do not shift markedly from historical patterns. This is the business-as-usual scenario.

- SSP3 - A Rocky Road (High challenges to mitigation and adaptation): Resurgent nationalism, concerns about competitiveness and security, and regional conflicts push countries to increasingly focus on domestic or at most, regional issues, which results in a lower GWP trajectory than the business-as-usual (i.e., SSP2) scenario.

The SSP scenarios already contain population trajectories that we are not going to use as inputs, but that are worth describing. Figure 4a shows that the population assumed in SSP1 is really closed to the UN low variant, whereas population in SSP2 (respectively SSP3) lies in between the UN medium and low (medium and low) variants. Figure 4b gives a visual representation of the three ever-increasing exogenous GWP trajectories that we used as inputs for our world population model, while Figure 5 and Figure 6 show the simulation results. 


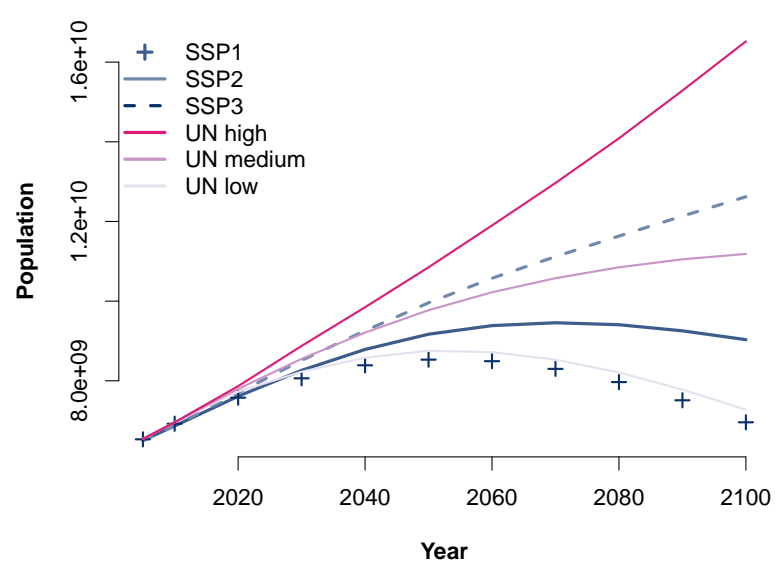

(a)

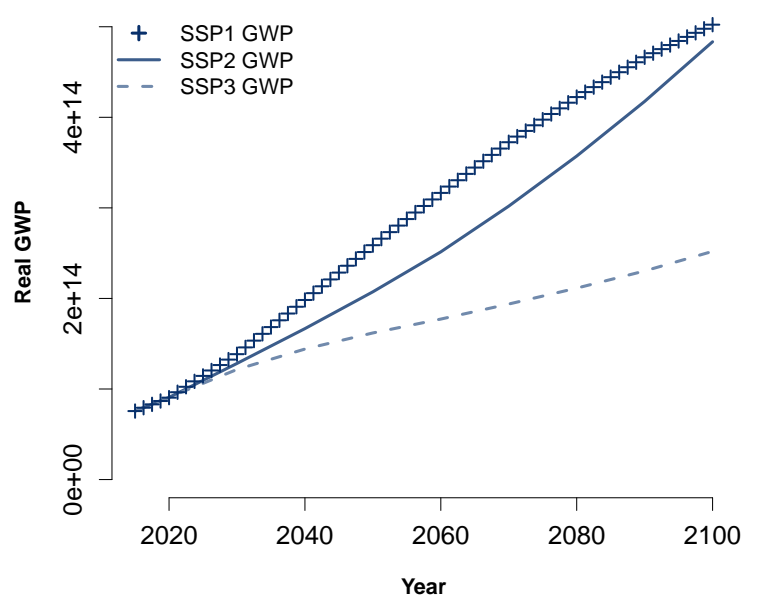

(b)

Fig. 4. (a) original population trajectories of SSP scenarios compared to United Nations variants, and (b) Three scenarios of exogenous ever-increasing GWP, 2015-2100.

\subsubsection{Populations projections with exogenous ever-increasing GWP scenarios}

The most striking result from our simulation is that given the significant differences between the GWP input of the three SSP scenarios, and contrary to the original population trajectories embedded in these scenarios (Figure 4a), there is notable consistency in the population dynamics delivered by our model for the SSP1 GWP, SSP2 GWP, and SSP3 GWP scenarios. As shown in Figure 5, simulations under the three different exogenous GWP from SSP scenarios are indeed extremely similar and very close to the United Nations medium projection up to 2065, after which date they are clearly above. A corollary from this main result is that in the low and medium variants of the UN population model, fertility rates decrease far more rapidly after 2065 than in the previous decades of the twenty-first century.

Given our model, this behavior makes perfectly sense. Indeed, although we consider different exogenous GWP dynamics in each of the three SSP scenarios, all of them consider GWP levels that are above the 2015 level. Hence, in all our simulations using the GWP from one of the three SSPs, prevailing birth rate and death rates correspond to the lower asymptotic limits of Figure $1 \mathrm{~b}$, and Figures $2 \mathrm{a}$ to $2 \mathrm{~d} .{ }^{13}$ Our simulation results yield two major implication. First, the SSP scenarios developed for the last Edenhofer et al.'s (2014) report of Working Group III take no account of the historical relationship that prevailed between GWP and population from 1950 to 2015. In other words, the population trajectories assumed in SSP scenarios (shown in Figure 4a) are uncorrelated to the GWP trajectories of these same scenarios. Second, the low and even medium prospective variants of the United Nations also assume future decoupling possibilities between economic development and fertility that have never been witnessed during the last sixty-five years.

\footnotetext{
${ }^{13}$ Another sensitivity analysis of the model under scenario SSP2 is provided in Figure III.1 of Appendix III using ad-hoc birth and death rates.
} 
That being said, because our model was calibrated on historical ever-increasing GWP, we can be more confident in the output it delivers under prospective ever-increasing GWP trajectories (such as the three SSP scenarios). But what about a context of long-lasting economic degrowth? Moreover, if our human population model is to be included in IAMs, the impacts of climate change on population dynamics should also be considered. As discussed in the following section, economic collapse and climate change constitute two (possibly associated) regime changes that may imply some refinements of our world population model.

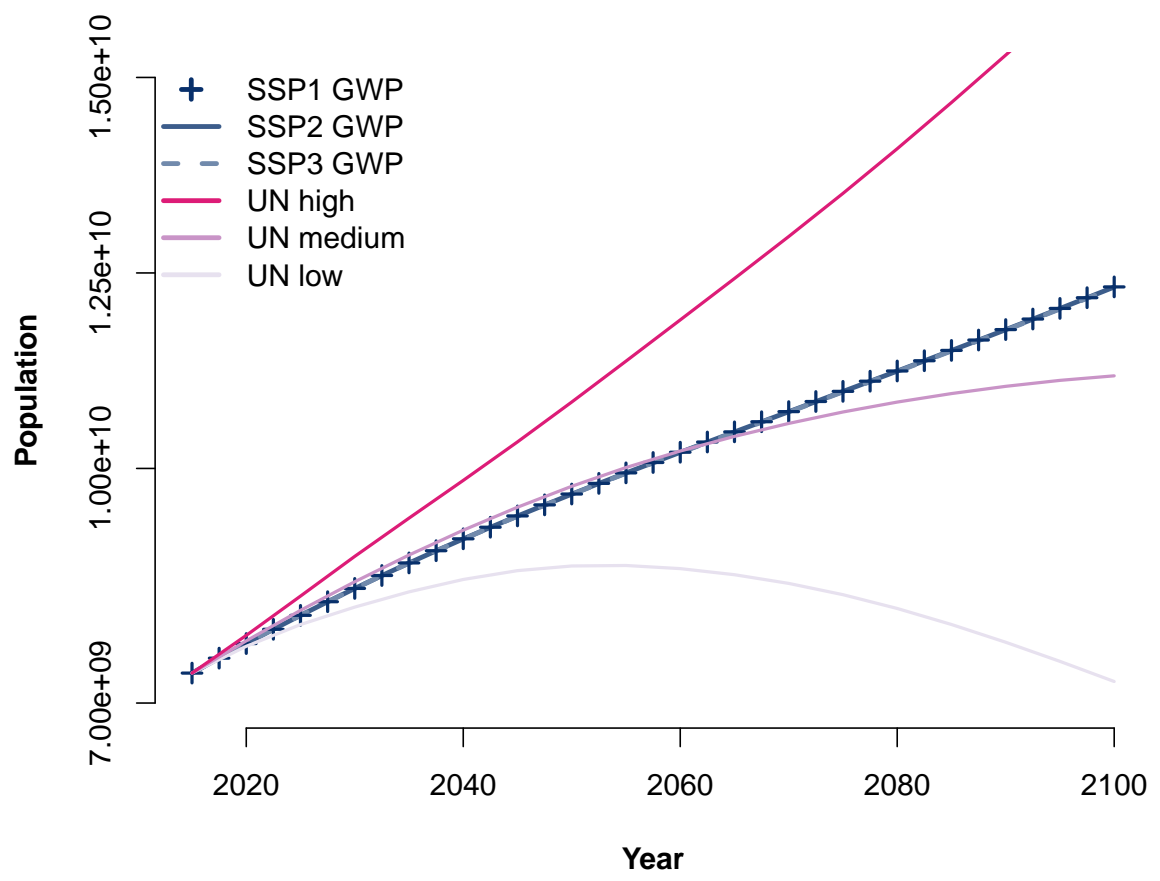

Fig. 5. Global population projections of the model for the three exogenous GWP scenarios compared to three United Nations variants, 2015-2100.

\section{Discussion: economic degrowth and climate change}

In this section, we discuss two possible refinements of our model, namely the impact on birth and deaths rates of economic degrowth events (5.1), and climate change (5.2).

\subsection{Investigating regime change under economic degrowth}

\subsubsection{What happens in our model in a Collapse scenario?}

Let us first look at our model outcome under an exogenous future scenario of drastic economic degrowth: 


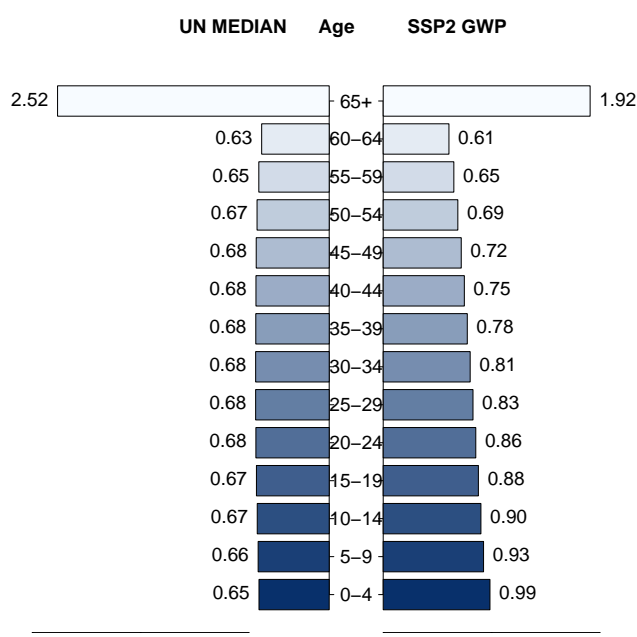

(a)

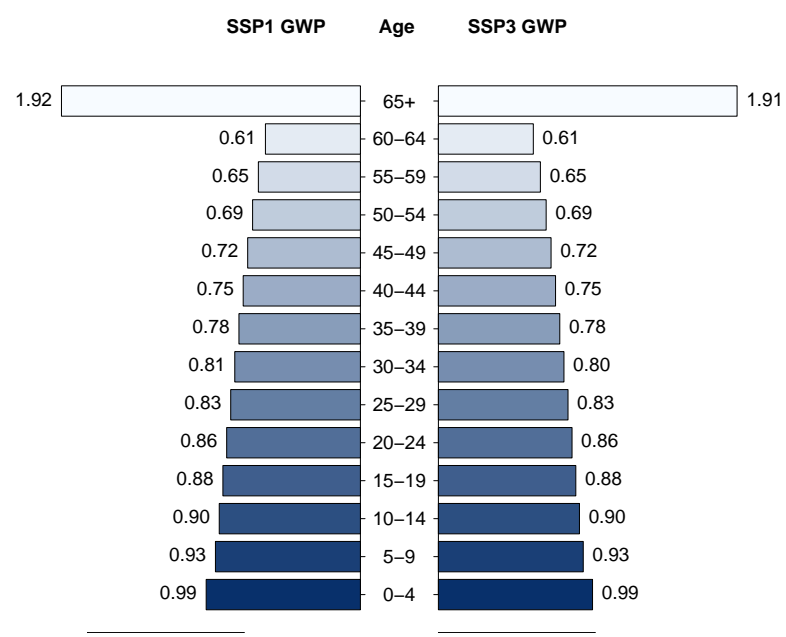

(b)

Fig. 6. Population pyramids in $\mathbf{2 1 0 0}$ for the UN median projection and the three prospective simulations of our model with ever-increasing GWP scenarios (unit is billion).

- Collapse - Going Down the Slope (Abrupt economic collapse in the middle of the twenty-first century): assumes the SSP2 scenario until 2050, then over five years GWP linearly decreases to one half of the GWP in 2015, because of an assumed major financial or environmental crisis.

Running the Collapse GWP scenario yields very different results in comparison with the outputs of the three SSP scenarios. The most noticeable result is that once drastic economic degrowth starts, population dynamics increasingly diverge upwards (Figure 7a). The abrupt collapse implies that the birth rate increases relatively to the death rates so that the overall population grows and gets younger. The age structure of the population in the Collapse GWP simulation is indeed significantly different from the three SSP scenarios (Figure 7b). As illustrated in Figure 8, this difference in age structure is important in terms of active population size and labor force availability. In 2075 (2100 respectively) the active population is 6 (4.4) percentage points lower in the Collapse simulation relative to the SSP2 scenario.

The main result of an increasing population in the Collapse scenario may appear counterintuitive to some readers who would more willingly imagine a sharp decrease in the population level as, for instance, in the World3 model of Meadows et al. (1972). However, in the World3 model it is the over-exploitation of non-renewable resources, together with the resulting unsustainable levels of pollution, that leads to a decrease in soil fertility and, consequently, to a decrease in agricultural production, and a subsequent decrease in population. Conversely, in the present paper we are not modelling agricultural production, but rather are pointing out that given the relationships that have prevailed for the past 65 years between the GWP per capita on the one hand, and birth and death rates on the other hand, a Collapse scenario implies an increase and not a decrease in the world population. This is because some kind of "ergodicity hypothesis" underlies our approach: history has witnessed ever-increasing GWP together with ever-increasing global population (with few 


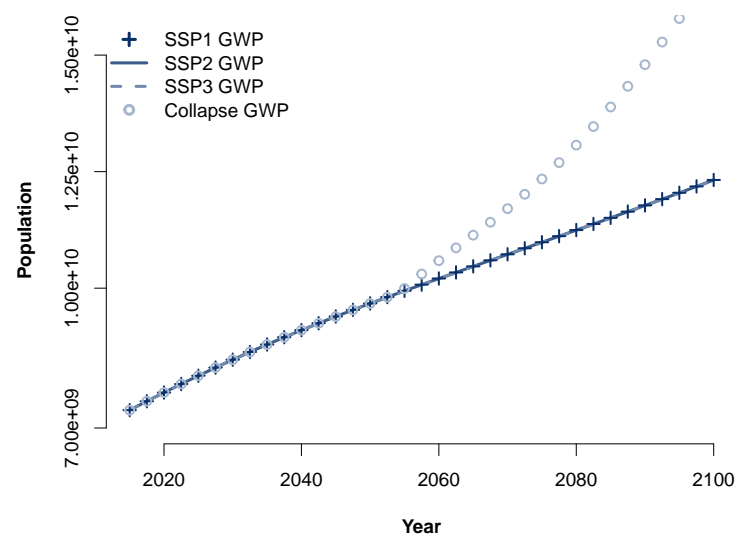

(a)

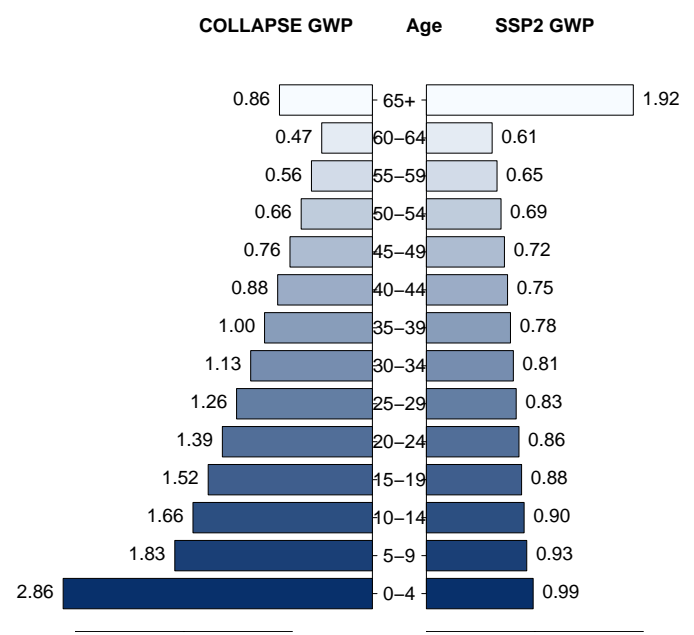

(b)

Fig. 7. (a) Global population projections of the model for the four exogenous GWP scenarios compared to three United Nations variants, 2015-2100; (b) Population pyramids in 2100 for the SSP2 and Collapse projections (unit is billion).

local exceptions), therefore, the historically-calibrated functional relationship we defined in Section 3.2 between world population and GWP (eluding the monotonous direction of co-variables) assume that the return to a lower level of GWP implies a turning back to previous birth/death rates. One might argue that our model is wrong and should take into account an "hysteresis hypothesis" such that an abrupt economic collapse would not be associated with a return to previous birth/death rates mostly because knowledge, and to a certain extent technical level associated with it, is maintained even in case of drastic economic degrowth. If one adopts such an approach, our model needs to be amended. In order to assess the possibility of introducing asymmetry into our model with an economic degrowth regime, we review the impacts of past economic recessions on birth and death rates. Although our model takes a global perspective, we investigate the impact of recessions at the country level. We are well aware of the potentially spurious conclusions that can arise when mixing assessments of such disparate scales (e.g., aggregation problem, emergence phenomenon). However, we are limited by the lack of global observations on the effects of deep economic recessions.

\subsubsection{The impact of economic recessions on birth and death rates}

The literature is not unanimous on the impact of economic recessions on fertility. However, most studies suggest that fertility trends often react pro-cyclically, with delays. Hence, periods of economic recession (or stagnation) are frequently followed within one or two years by a decline in birth rates. However, Sobotka et al. (2011) stress that these cyclical waves are usually short and relatively small (a few percentage points). The change can therefore be overshadowed by long-term secular trends in fertility caused by factors other than economic recession. This observation explains why a number of studies on fertility during the Great Depression of the 1930s-and other major recessionary events-found no statis- 


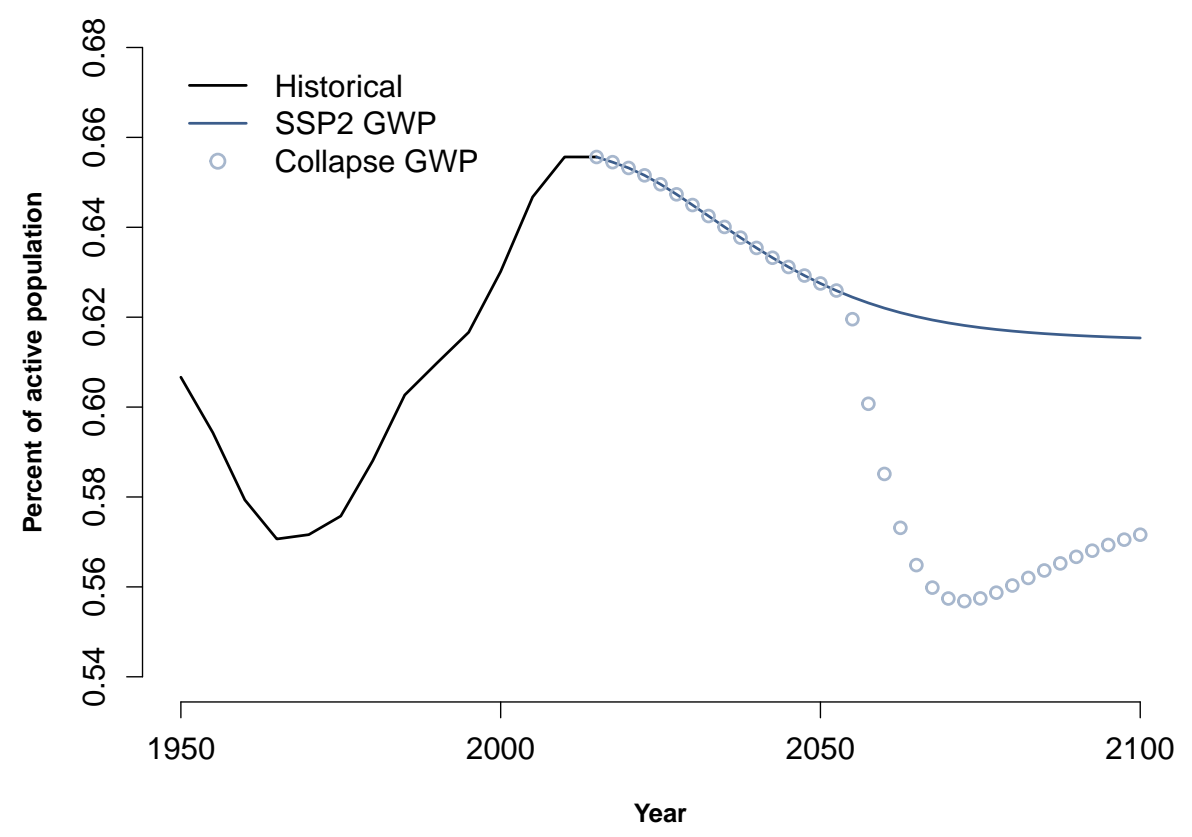

Fig. 8. Percent of active population: historical, 1950-2015; SSP2, and Collapse, 2015-2100.

tically significant relationship between the recession and the changes in birth rates. Both periods showed consistent long-term fertility declines that started well before recessionary events. Therefore, according to the evidence summarised in Table IV.1 (of Appendix IV), it is impossible to attribute a declining fertility trend to the recession itself, because in all countries that have be studied, birth rates were on long-term declining trends prior to degrowth episodes.

A significant corpus of articles shows that in developed countries general mortality and age-specific death rates tend to increase during economic expansions and decrease during recessions for both males and females-although the effect is more apparent for males. Hence, Tapia Granados and Diez Roux (2009) found that population health actually improved during the four years of the US Great Depression (1930-1933) with decreases in mortality for almost all ages, and increases of several years in life expectancy for both males and females, and whites and nonwhites. Similarly, Tapia Granados and Ionides (2017) show that in the European countries in which the Great Recession of 2007-2008 was particularly severe, mortality reductions in 2007-2010 were considerably larger than in 2004-2007. Broadly speaking, these studies find that during episodes of economic crises, deaths due to infectious disease and transport accidents tend to decrease, while deaths caused by diabetes, hypertensive disease, chronic poisoning (alcohol, tobacco), and suicide tend to increase to a lesser extent. The result is a decrease in the aggregate death rate. In other words, in these examples, the detrimental effect of recessions on health is not important enough to revert the accumulated progress of the health system. However, the dissolution of the Soviet Union offers a counter-example of countries facing prolonged economic degrowth associated with an increased death rate (see Shkolnikov et al. (2001) for Russia, and Carlson and Tsvetarsky (2000) for Bulgaria). Hence, the evidence summarised 
in Table IV.1 (of Appendix IV) suggests that economic recessions must be severe enough, and associated with a collapse of political institutions - at least partially -, in order to observe an increase in the death rate during an economic crisis (for former USSR countries) or just after (for Greece, Germany, and possibly Venezuela). If political institutions are not too severely disturbed during recessions, such that material needs and health infrastructures hold, then death rates seem to stay on their declining pace (Argentina, Mexico, USA, Peru, Chile, Zimbabwe).

In summary, it seems warranted to consider that it is the level of GWP per capita, rather than its upward or downward change, that determine both the birth rate and death rates of population. As a consequence, the global population model presented in Section 3 does not require further adjustments. Even if it seems counter-intuitive, an increasing population in case of abrupt economic collapse is quite possible. Knowledge and technical levels would not vanish instantly in case of economic collapse, but the drastic decrease in GWP per capita that would come with such an event implies that both economic and political institutions and people's behaviors would be heavily disrupted, to the extent that aggregate birth/death rates could indeed return to values characterizing past lower levels of economic development. Nevertheless, concerns from the scientific community indicate that future death rates could be significantly altered by climate change (possibly in association with economic degrowth). In the following section, we thus turn to an extension of our model that considers climate change feedback on death rates.

\subsection{Excess mortality function associated with climate change}

Most IAMs take into account the impacts of climate change through a damage function on production, capital, or productivity (Diaz and Moore, 2018; Tol, 2018), while the population dynamics follow an exogenous trend. We argue that endogenizing the population dynamics would require a distinct damage function that would include the idiosyncratic effect of climate change on human health.

\subsubsection{Framework}

Climate change affects human health in four principal ways, namely, (i) increased undernutrition due to decreases in crop yields, and increased malnutrition due to changes in the macro- and micro-nutrients contents of cereals (Smith and Myers, 2018; Springmann et al., 2016), (ii) altered distribution of allergens and vector-borne infectious diseases resulting in higher risk of typhus, cholera, malaria, dengue, and West Nile virus infection (Franchini and Mannucci, 2015), (iii) increased prevalence of diarrhoeal diseases due to more frequent and longer drought periods causing reduced safe water availability (Kolstad and Johansson, 2011), and (iv) increased frequency of heat waves that translates into higher mortality related to acute and chronic respiratory, or cardiovascular diseases (Mora et al., 2017). ${ }^{14}$

\footnotetext{
${ }^{14}$ At mid latitudes increasing temperature may reduce the rate of diseases related to cold temperatures (such as pneumonia, bronchitis and arthritis), but these benefits are unlikely to counterbalance the global risks associated with warming that low latitudes regions will suffer the most (Gasparrini et al., 2017).
} 
The 2014 assessment performed by the World Health Organization (WHO, 2014) is, to the best of our knowledge, the only study estimating the total number of additional deaths that can be attributed to climate change at the global scale. The assessment uses an IPCC's (2000) A1b scenario, for 2030 and 2050, and for five mortality risks: undernutrition (for children under 5), malaria (for all ages), dengue (for all ages), diarrhoeal diseases (for children under 15), and heat waves (for people above 65). For undernutrition, malaria, and diarrhoeal diseases, the global additional number of deaths estimated in the WHO (2014) study are higher in 2030 than in 2050 (see Table V.1 in Appendix V). This counterintuitive result is due to the assumed adaptation of population to climate-induced mortality risks; however, assumed adaptation is not sufficient to prevent an increase in heat-related and dengue-related deaths between 2030 and 2050.

As far as we are aware, Pottier et al. (2018) have suggested the first excess mortality function associated with climate change. In their framework, each of the five climateinduced mortality risks identified in the WHO (2014) study constitutes an element $j$ among the set $J=$ \{Undernutrition, Malaria, Dengue, Diarrhoeal diseases, Heat waves $\}$. As shown in Eq. 6, the excess mortality function associated with climate change consists of a multiplier that increases the specific death rate of any age-group $i$ in the population model,

$$
\widetilde{D R_{i}}=D R_{i} \underbrace{\left[1+\sum_{j \in J} \alpha_{i, j}\left(\frac{T}{T^{0}}\right)^{\theta}\right]}_{\text {Climate change induced multiplier }} .
$$

Where $\alpha_{i, j}$ is the relative increase in the probability of dying due to risk $j$ for the age-group $i$ at the calibration temperature increase $T^{0}$ (i.e., the temperature change of $+2.5^{\circ} \mathrm{C}$ in 2050 for the A1b scenario used in the WHO (2014) study), $T$ is the prevailing global temperature change (also called temperature anomaly) relatively to the pre-industrial era, and parameter $\theta$ specifies the dependence of the probability of dying with respect to temperature.

As discussed in Pottier et al.'s (2018) article, four main assumptions are embedded in the choice of the functional form of Eq. 6. First, the climate-induced death rates are multiplied by the death rate without climate change. This is a reasonable assumption, since climate-induced mortality will certainly be influenced by general sanitary conditions, health systems, and the availability of pharmaceutical drugs, which are all factors that are already reflected in the evolution of the $D R_{i}$ over time. Second, the different $j$ risks do not equivalently affect the different $i$ age-groups as we follow the WHO (2014) classification (e.g., malaria affects all age groups, whereas diarrhoeal diseases only affect children under 15). However, the overall number of additional deaths associated with a given climateinduced risk $j$ are evenly distributed over the $i$ age-groups that are specifically affected by such a risk. So, in 2050 for instance, the 32,695 malaria-induced additional deaths are evenly distributed over the 14 different age groups, whereas the 32,955 diarrhoealinduced additional deaths are evenly distributed over the first three age groups of the population. Third, a given mortality risk increases with climate change without taking into account adaptation, which means that our modeling choice cannot comply with the non-monotonicity over time that the WHO (2014) study estimates for deaths caused by undernutrition, malaria, and diarrhoeal diseases (see Table V.1 in Appendix V). Fourth, the 
global temperature increase (or anomaly) is used as a proxy for climate change. This is a crude assumption, as mortality is more likely to depend on more specific climate data such as precipitation or moisture. However, this assumption logically follows the equivalent modeling choice made in both the WHO (2014) study and in most IAMs. Finally, Pottier et al. (2018) have chosen a power-dependency for temperature, akin to the usual damage functions used in climate change economics literature. This power-dependency, $\theta$, the probability of dying with respect to temperature, is the same for each risk, meaning that it does not depend on a given risk $j$. The sensitivity of the results to this parameter will be tested. Due to space constraints, the calibration procedure of parameters $\alpha_{i, j}$ is detailed in Appendix V.

\subsubsection{Simulations}

Given the uncertainty surrounding the WHO (2014) study, the simulations presented in this section should only be used to assess the sensitivity, not the outputs, of the excess mortality function due to climate change presented in Eq. 6. We simulated our model with the exogenous GWP of either the SSP2 or the Collapse scenario, together with three different temperature trajectories displayed in Figure 9. The names of the temperature scenarios $\left(+4^{\circ} \mathrm{C},+3^{\circ} \mathrm{C}\right.$, and $\left.+2^{\circ} \mathrm{C}\right)$ correspond to the temperature anomaly reached in 2100 compared to the pre-industrial era. Given the wide variability of the simulation results of climate models for the same greenhouse gas emission trajectory (i.e., representative concentration pathway, RCP, in the IPCC's lexicon), we did not use the outputs of a given climate model and chose instead to determine the temperature trajectories in Figure 9 so that they reflect some representative patterns of the IPCC's RCP.

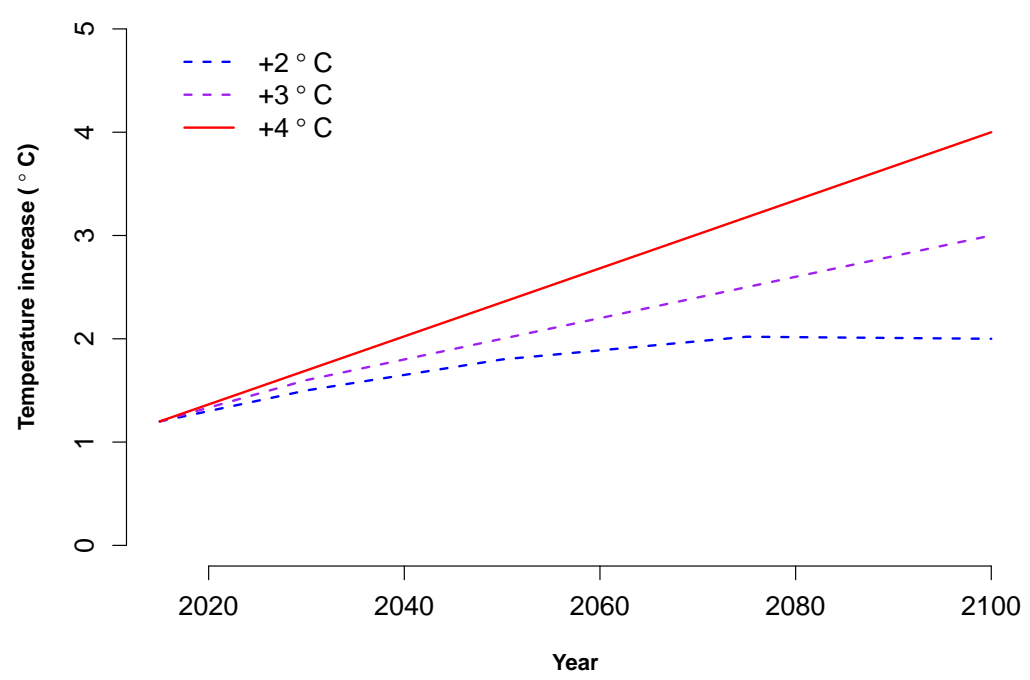

Fig. 9. Three prospective scenarios of temperature change (relatively to the pre-industrial era).

As expected, for a given exogenous GWP trajectory, the higher the temperature increase due to climate change, the lower the population. By calibration (see Appendix V), the 
number of additional deaths is quite similar across scenarios, and prior to 2050 the number of additional deaths remains below 500,000 per five-year period in each temperature scenario. However, in 2100, the $+4^{\circ}$ scenario implies about twice as many deaths per five-year period ( 2.5 millions) than in the $+2^{\circ} \mathrm{C}$ scenario ( 1.25 millions) when $\theta=1$, and logically around four times more (4 million compared to less than 1 million) when $\theta=2$ (Figure 10a and 10b). If the exogenous GWP of the Collapse scenario is used instead of SSP2, climate change logically generates a higher number of additional deaths as the total population-in particular the younger cohorts-is larger (Figure 10c and 10d). For example, in the $+2^{\circ} \mathrm{C}$ scenario when $\theta=2$, there are approximately 6 million additional deaths per year in 2100 in the Collapse scenario, compared to approximately 4 million in the SSP2 scenario. In order to further test the sensitivity of the excess mortality function associated with climate change, we performed the previous simulations again, replacing the WHO's (2014) estimate of 84,697 nutrition-related additional deaths per year in 2050 with the far higher Springmann et al. (2016) value of 381,000 additional deaths (see Table VI.1 and Figure VI.1 in Appendix VI).

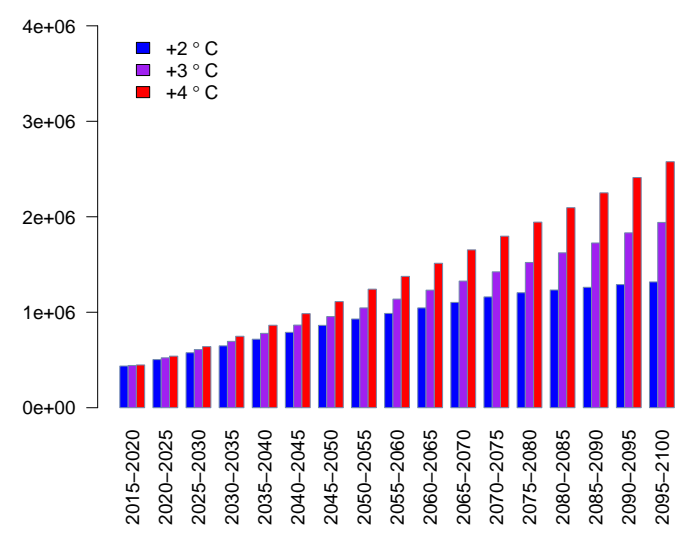

(a) $\theta=1$

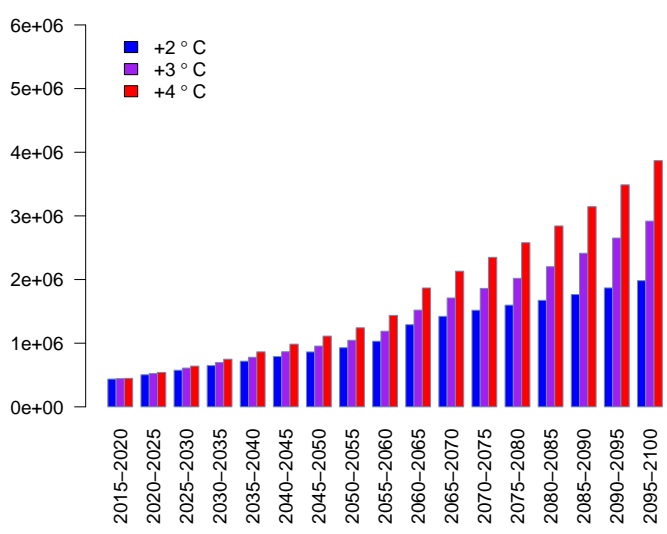

(c) $\theta=1$

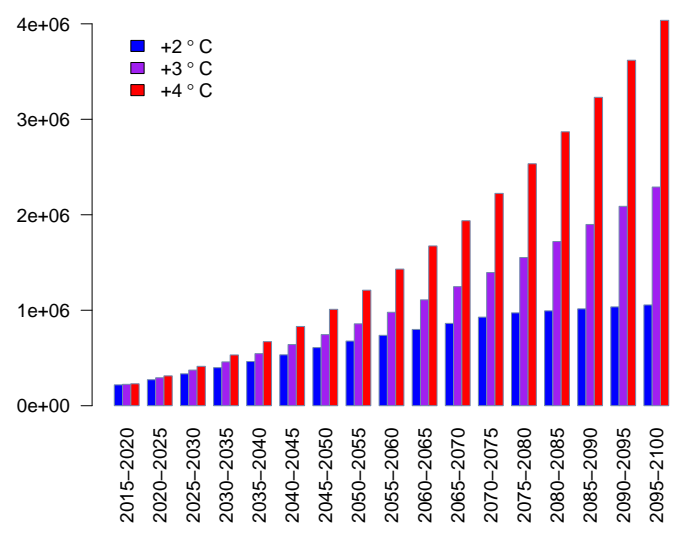

(b) $\theta=2$

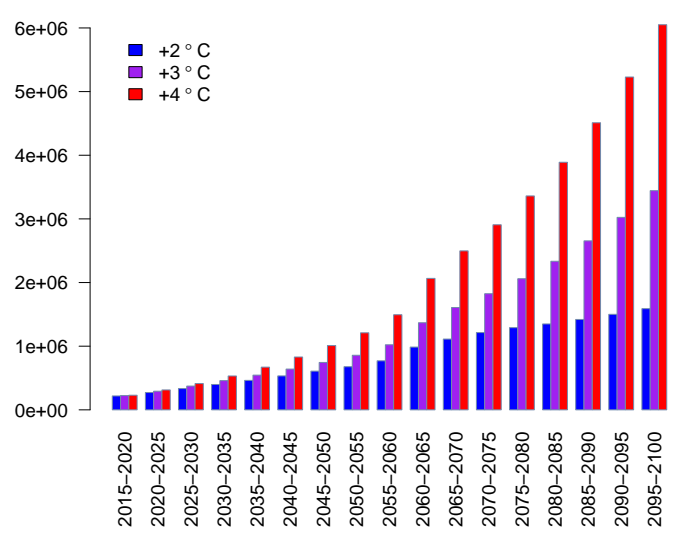

(d) $\theta=2$

Fig. 10. Climate change induced deaths every five years with exogenous (a-b) SSP2 and (c-d) Collapse GWP. 


\section{Conclusion}

Understanding demographic dynamics is undeniably complex because multiple interconnected factors affect both birth rates and death rates. In this article, we attempted to circumvent this complexity by providing a simple endogenous model of the world human population, with just enough complexity to present an age structure. Using the gross world product as the only exogenous input variable to determine the birth rate and age-specific death rates, we designed a phenomenological model of the global population dynamics.

The analysis of this theoretical model suggested that it is most likely asymptotically unstable, preventing its use in assessments of the carrying capacity of the Earth. Nevertheless, we showed that our model accurately reproduces the global population dynamics over the period 1950-2015. Moreover, for any prospective scenario of increasing GWP, the model presented in this paper yields consistent outputs. A major implication of this simulation result is that both the United Nations and the IPCC take no account of the historical relationship that prevailed between GWP and population from 1950 to 2015, and thus assume future decoupling possibilities between economic development and fertility that have never been witnessed during the last sixty-five years. On the contrary, in the case of an abrupt collapse of the economic production, the population dynamics of our model responds with higher deaths rates that are more than compensated for by increasing birth rates. Hence, in such a scenario of collapsing GWP, the population gets relatively larger and younger (i.e., there is an increase in the size of the $0-4$ years-old group relative to other age groups). As we noted, this result might appear counter-intuitive at first, in particular if one has in mind the simulation outputs of the World3 model of Meadows et al. (1972). Yet, we recalled that results in World3 are driven by negative feedback from the lack of non-renewable resources and unsustainable pollution levels, which combined food production to degrowth drastically.

Hence, we then discussed the implementation of environmental negative feedback in our model. In order to comply with existing Integrated Assessment Models, we investigated the sensitivity of our model to an excess mortality function associated with climate change. The calibration of the health-related damage function was performed using the most recent and consistent data from the WHO (2014). Even though it can only yield relative and not absolute outcomes, estimates of additional deaths in 2100 due to climate change range from 1 million per five-year period in a $+2^{\circ}$ scenario to 6 million per five-year period in a $+4^{\circ}$ scenario. These additional deaths caused by climate change hardly affect the world population level reached at the end of 2100 . This result could receive two very different interpretations: (i) the estimates of the WHO (2014) study are extremely conservative, or (ii) the results of Meadows et al. (1972) are highly unrealistic because they would imply that the WHO's (2014) estimates should be multiplied by a factor of 100 to 1000 .

We acknowledge that the climate change component of the model developed in the present paper is not robust. Nevertheless, this article is, to the best of our knowledge, the first to present a complete endogenous framework of world population dynamics that can both take into account the effects of climate change, and be used into any kind of IAM. We hope that future studies assessing the impact of climate change on additional deaths will be used to better calibrate our model and therefore improve its estimates of the impacts of 
climate change on human population dynamics.

\section{Appendices}

\section{Appendix I - Overpopulation: old concerns die hard}

Until approximately 300 years ago, world population growth has been very low, at around $0.04 \%$ per year, from four million people in 10,000 BCE to 610 millions in 1700 . However, the Industrial Revolution drastically changed this pattern of long-lasting limited growth, and global population reached 1.2 billion individuals by 1850 , which corresponds to an annual increase of $0.45 \%$ during the period 1700-1850 (Kremer, 1993, and Figure I.1). Several classical economists of the time raised concerns about such rapid population growth. By considering exponential growth for the population dynamics, while food production followed a linear increase due to decreasing returns on land, Malthus (1798) predicted that the English population would ultimately lack food supply, which could only result in unavoidable deaths by hunger and disease. ${ }^{15}$ This prediction proved to be erroneous because Malthus did not reckon the massive yields increases that took place in the agricultural sector at the time (Smil, 2017, p. 114) .

From 1850 to 1920 , the growth rate of the world population has slightly increased by about $0.55-0.60 \%$ per year, resulting in a global population of 1.8 billion. After 1920, another order of magnitude change can be observed. Exceeding 1\% in the 1940s, the annual growth rate of the wold population steadily increased and reached its peak at $2.2 \%$ in 1962-63 with a global population of 3.15 billion (Figure I.1). At this point, the fear of overpopulation came back in several scholars' writing and, exactly 170 years after Malthus, Ehrlich became famous for his (pessimistic) predictions in The Population Bomb (1968). Again, those forecasts of impending world famines proved inaccurate due to an increase in food production from the conversion of forests to agricultural lands (Goldewijk et al., 2017) and agricultural yield improvements (Smil, 2017, p. 312).

As of 2015, the world population was about 7.4 billion and its annual growth rate was approximately $1.15 \%$. Considering the United Nations's (2017b) medium projection of a growth rate reaching $0.09 \%$ per year at the end of this century, the global population would be just below 11.2 billion in 2100 (Figure I.1). Accordingly, in November 2017, the concern about overpopulation was renewed in a statement of 15364 scientists from 184 countries who indicated that humanity is jeopardizing its future by not reining in its "intense but geographically and demographically uneven material consumption and by not perceiving continued rapid population growth as a primary driver behind many ecological and even societal threats" (Ripple et al., 2017, p. 1026). The strong warning of Ripple et al. (2017) ends with thirteen proposed steps that mankind should undertake to transition towards a more environmentally sustainable alternative to the so-called business as usual. Among them, Ripple et al. (2017, p. 1028) suggest "estimating a scientifically defensible,

\footnotetext{
${ }^{15}$ This initial formulation made Malthus both famous and infamous, but in later life he took a much more nuanced attitude to the balance between production and population. In particular, Malthus came to appreciate that nuptiality played a very important role in determining population trends, influencing both fertility levels, and indirectly, also mortality levels (Wrigley, 2016, pp. 24-25).
} 
sustainable human population size for the long term while rallying nations and leaders to support that vital goal".

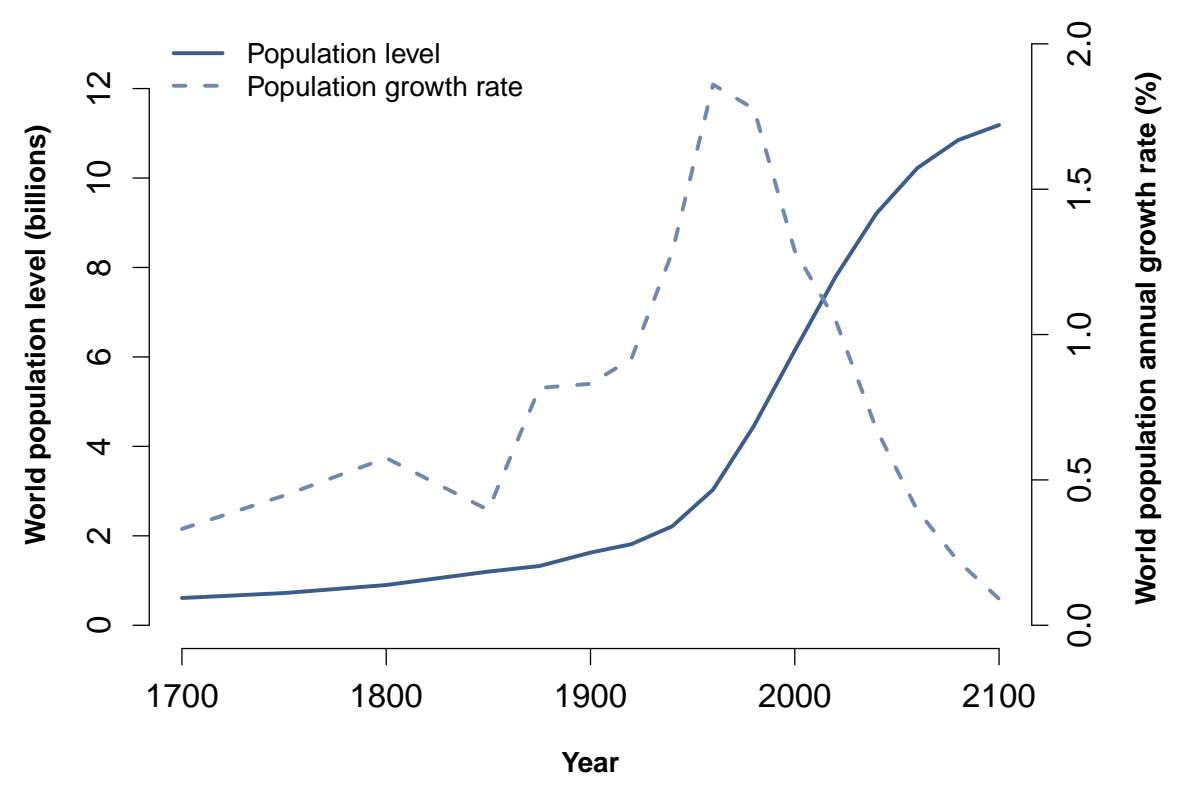

Fig. I.1. Historical and projected world population level (left axis) and annual growth rate (right axis), 1700-2100. Data source: Kremer (1993) from 1700 to 1950, United Nations's (2017b) medium estimate from 1950 to 2100.

Of course, for a given level of pollution, it is not the population level per se that represents a problem, but rather a combination of population with the level of per capita consumption, and the technical level defining the pollution by unit of consumption. ${ }^{16}$ Since technical change is not more foreseeable than before, one could argue that, once again, future innovations are going to alleviate the human population burden on Earth in the coming decades. For technical optimists, the current overpopulation concern will add to the list of failed overpopulation predictions of the past. However, no one can deny that food supply is heavily supported by the use of fossil fuels, either directly through fertilizers and mechanization, but also indirectly through the general use of transport, industry, and services (Smil, 2017, pp. 306-313). Harchaoui and Chatzimpiros (2018) even suggests that modern agriculture hardly generates net energy surpluses, which means that an energy transition from fossil fuels that would not be compensated by enough net energy from renewable energy could jeopardize global agricultural production, and thus world population.

\footnotetext{
${ }^{16}$ This controversy is well illustrated by the $I=P A T$ equation, where $I$ is the impact of human activity on the environment (i.e., the pollution level), $P$ is the population level, $A$ stands for affluence (i.e., the level of per capita consumption), and $T$ represents the technical level. See Chertow (2000) for an historical analysis of the various forms the IPAT equation has taken since its formulation in the 1970s.
} 


\section{Appendix II - Best-fit parameters of the global birth rate and death rates sigmoid functions of our model}

Table II.1: Best-fit parameters of the global birth rate sigmoid function.

\begin{tabular}{ccccc}
\hline$\overline{\mathbf{B R}}$ & $\underline{\mathrm{BR}}$ & $\delta$ & $\phi$ & $\nu$ \\
\hline 0.39 & 0.18 & 0.00087 & 4033.6 & 0.18 \\
\hline
\end{tabular}

Table II.2: Best-fit parameters of the global death rate sigmoid function for each 5-year age group.

\begin{tabular}{cccccc}
\hline Age group & $\overline{\mathbf{D R}_{\mathbf{i}}}$ & $\underline{\mathbf{D R}_{\mathbf{i}}}$ & $\delta_{\mathbf{i}}$ & $\phi_{\mathbf{i}}$ & $\nu_{\mathbf{i}}$ \\
\hline$N_{1}$ & 0.60192 & 0.0392421 & 0.00051169 & 3951.5 & 1.76828 \\
$N_{2}$ & 0.34980 & 0.0045840 & 0.00054322 & 3441.8 & 11.99152 \\
$N_{3}$ & 0.33501 & 0.0042607 & 0.00058728 & 3312.8 & 15.02963 \\
$N_{4}$ & 0.33232 & 0.0058662 & 0.00063047 & 3282.7 & 15.63468 \\
$N_{5}$ & 0.33982 & 0.0085766 & 0.00066358 & 3364.8 & 14.48400 \\
$N_{6}$ & 0.34346 & 0.0100244 & 0.00069397 & 3323.4 & 12.63958 \\
$N_{7}$ & 0.32770 & 0.0117765 & 0.00072698 & 3297.5 & 10.48483 \\
$N_{8}$ & 0.35451 & 0.0135612 & 0.00064659 & 3469.5 & 10.33744 \\
$N_{9}$ & 0.34216 & 0.0168843 & 0.00060129 & 3365.7 & 7.43185 \\
$N_{10}$ & 0.35791 & 0.0224452 & 0.00054379 & 3601.1 & 6.59593 \\
$N_{11}$ & 0.38159 & 0.0327278 & 0.00052461 & 4018.9 & 6.23736 \\
$N_{12}$ & 0.39385 & 0.0467704 & 0.00048987 & 4187.2 & 4.28997 \\
$N_{13}$ & 0.39473 & 0.0721157 & 0.00048556 & 4246.3 & 2.64664 \\
$N_{14}$ & 0.49206 & 0.2701185 & 0.00054744 & 4104.9 & 0.85705 \\
\hline
\end{tabular}




\section{Appendix III - Model sensitivity to changes in the death rate of the fragile population and propagation erors}

To test the sensitivity of the model without climate change, we modify the death rate of the fragile population, defined as the first and the last groups (i.e., $N_{1}$ and $N_{14}$ ), ceteris paribus in 2100 (i.e., keeping the GWP from the SSP2 scenario and the birth rate constant). The results are displayed on the heatmap of Figure III.1. For instance, this figure indicates that in order to observe a global population of about 4.5 billion people in 2100, as found in the standard run of the (in)famous study of Meadows et al. (1972), the death rate of group $N_{1}$ ( $0-4$ years old) would have to be multiplied by about fifteen.

We note that an increase in the global mortality rate of $N_{1}$ mimics the dynamics a similar decrease in $B R$ for all age groups, except for the first age group that would be overestimated. Therefore, model sensitivity to birth rate would lead to similar results of Figure III.1 although $N_{1}$, which is relatively small with respect to the sum of all other age groups, will be lower than the one used to construct the heatmap.

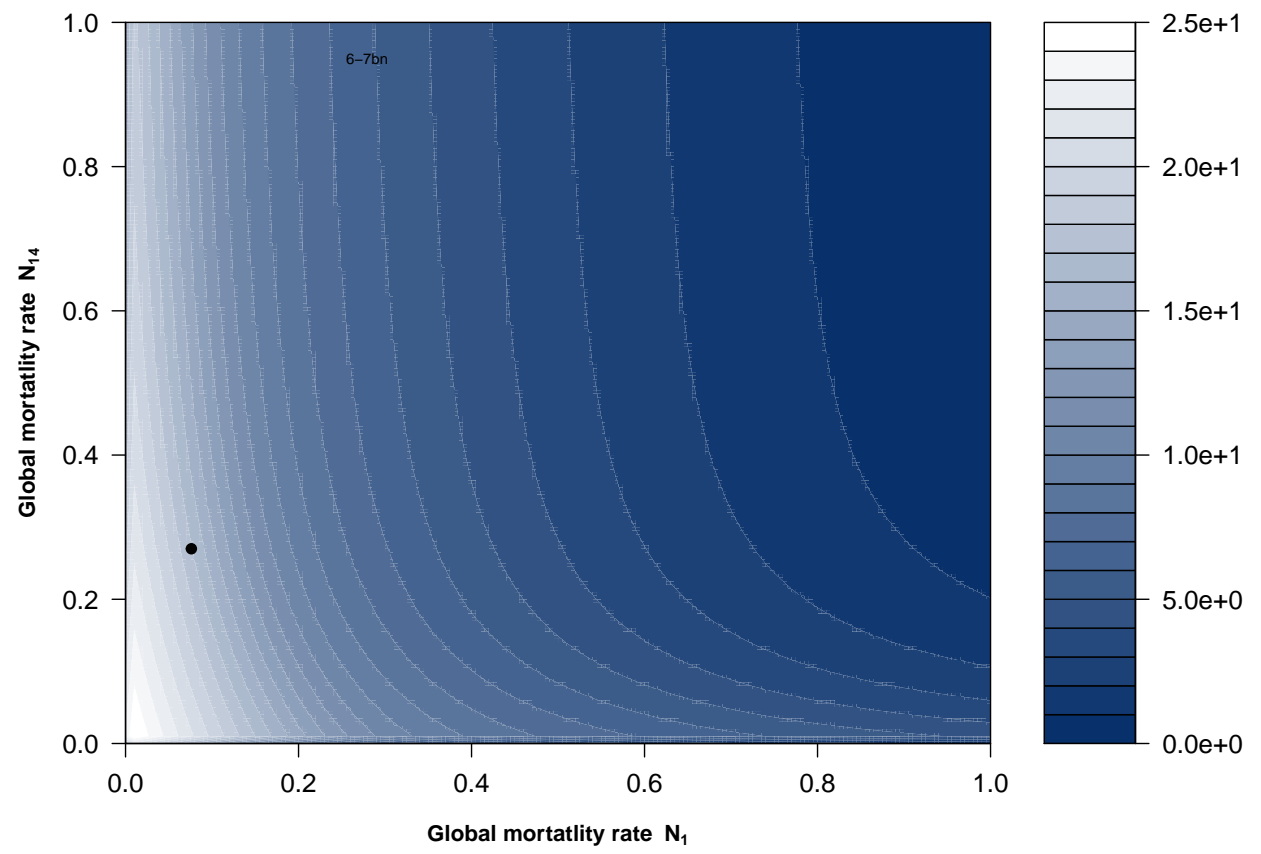

Fig. III.1. Sensitivity of global population level in 2100 as a function of groups $N_{1}$ (0-4 years old) and $N_{14}$ (+65 years old) specific death rates $D_{1}$ and $D_{14}$. The black dot represents the population of about 12 billion attained in the SSP2 GWP scenario.

To test propagation errors of the model due to wrongly assessed initial conditions, we add one member per age group, then we run the simulation ceteris paribus until 2100 and report the total addition in population with GWP from the SSP2 scenario. The results are shown in the graph of Figure III.2. For instance, this graph indicated that if the the cohort 4 is wrongly assessed by one unit, the difference in total population at the end of the century will be roughly three units. In other words, if cohort four happens to have one additional million people than the original simulation, the world will be populated by an additional 
three million individuals in 2100, which is a total increase of population of about $0.0083 \%$ with respect to the original results.

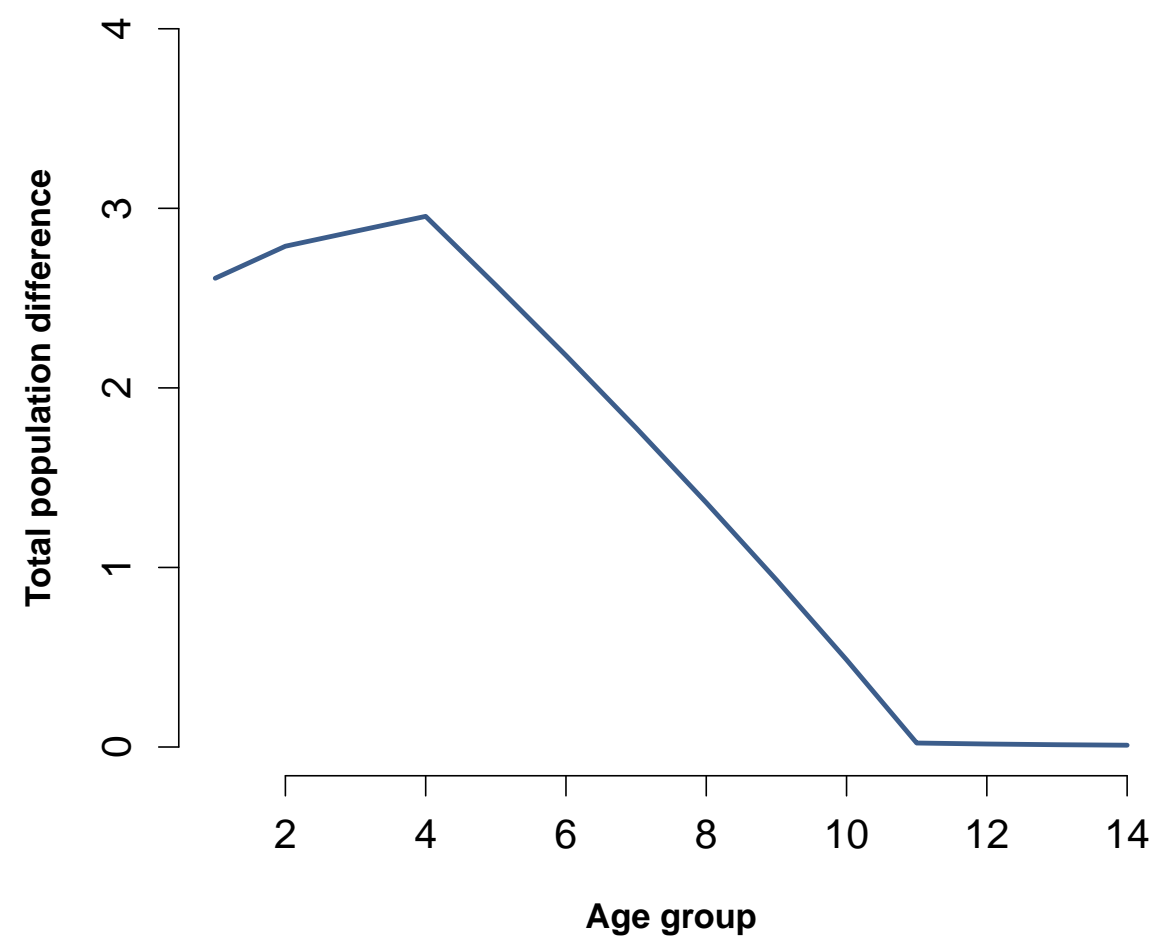

Fig. III.2. Total population difference in 2100 for an additional unit of a age group at in 2015 


\section{Appendix IV - Impacts of economic recessions on birth rate and death rate}

Table IV.1: Impacts of economic recessions on birth rate (BR) and death rate (DR).

\begin{tabular}{lccc}
\hline $\begin{array}{l}\text { Country (recession } \\
\text { time period) }\end{array}$ & $\begin{array}{l}\text { Avg. GDP/capita decline } \\
\text { during recession (\%/yr) }\end{array}$ & $\begin{array}{l}\text { Avg. change of BR before/ } \\
\text { during/after recession (\%/yr) }\end{array}$ & $\begin{array}{l}\text { Avg. change of DR before/ } \\
\text { during/after recession (\%/yr) }\end{array}$ \\
\hline Germany (1929-1932) & -4.7 & $-0.3 /-5.0 / 0.4$ & $-3.1 /-1.5 / 1.1$ \\
USA (1930-1933) & -8.8 & $-3.2 /-2.7 /-0.2$ & $? /-2.5 / 1.8$ \\
Chile (1972-1975) & -6.2 & $-2.1 /-3.1 /-1.0$ & $-2.7 /-3.5 /-2.6$ \\
Peru (1976-1978) & -2.2 & $-1.5 /-1.5 /-1.9$ & $-3.5 /-3.2 /-3.3$ \\
Peru (1988-1992) & -10.4 & $-1.9 /-1.8 /-2.2$ & $-3.3 /-2.6 /-2.1$ \\
Argentina (1988-1990) & -4.1 & $-1.3 /-0.7 /-1.3$ & $-0.6 /-0.5 /-0.6$ \\
Argentina (1999-2002) & -5.9 & $-1.3 /-0.7 /-0.7$ & $-0.6 /-0.2 /-0.2$ \\
Mexico (1986-1988) & -2.1 & $-2.8 /-1.5 /-1.6$ & $-3.3 /-2.0 /-1.7$ \\
Russia (1990-1996) & -7.4 & $-0.7 /-6.7 / 1.6$ & $-0.0 / 4.4 / 0.8$ \\
Bulgaria (1990-1998) & -1.2 & $-1.9 /-5.0 / 2.3$ & $1.2 / 2.0 / 0.4$ \\
Bulgaria (1999-2015) & -0.8 & $-4.8 /-4.6 / ?$ & $1.8 /-5.1 /$ \\
Zimbabwe (1999-2008) & -6.6 & $-1.5 / 0.6 /-0.7$ & $5.2 /-0.9 /-6.8$ \\
Greece (2007-2013) & -4.8 & $1.2 /-2.5 /-1.2$ & $0.8 / 0.5 / 4.8$ \\
Venezuela (2014-2016) & -10.5 & $-1.4 /-1.5 / ?$ & $0.9 / 0.8 / ?$ \\
\hline
\end{tabular}

Note: when data is available, average change of birth rate (respectively death rate) before and after a recessionary episode are calculated for the ten previous (respectively following) years.

\section{Appendix V - Calibration of excess mortality function associated with climate change}

To calibrate the $\alpha_{i, j}$, we borrow the methodology of Pottier et al. (2018). We note that calibrating the $\beta_{i}:=\sum_{j \in J} \alpha_{i, j}$ is sufficient to have the correct assessment of $\widetilde{D R_{i}}$ without identifying each elements of the set $J$ for a given age-group. We start by simulating the model without climate feedback under SSP2 scenario, and compute the number of deaths between 2045-2050 for each group. Then, assuming that the increase in temperature $T$ within this period equals $T^{0}$ (i.e., the temperature change of $+2.5^{\circ} \mathrm{C}$ in 2050 for the $\mathrm{A} 1 \mathrm{~b}$ used in the WHO (2014) study), we add the number of death induced by climate change in 2050 estimated by the WHO (2014, p.12) study (Table V.1). As the number of deaths induced by climate change is not evenly distributed over the age-groups, we assume the distribution provided by the WHO (2014) study and reproduced in Table V.2. Finally, Table V.3 provides the fitted values for parameters $\beta_{i}$.

Table V.1: Global additional deaths in 2030 and 2050 attributable to climate change for five mortality risks. Source: WHO (2014, p. 7 and p. 12, respectively).

\begin{tabular}{|c|c|c|c|c|c|c|}
\hline & Undernutrition & Malaria & Dengue & Diarrhoeal diseases & Heat waves & Total \\
\hline $\begin{array}{l}\text { Global additional } \\
\text { deaths in } 2030\end{array}$ & $\begin{array}{c}95,176 \\
(-119,807 \text { to } 310,156)\end{array}$ & $\begin{array}{c}60,091 \\
(37,608 \text { to } 117,001)\end{array}$ & $\begin{array}{c}258 \\
\text { (136 to } 331)\end{array}$ & $\begin{array}{c}48,114 \\
(21,097 \text { to } 67,702)\end{array}$ & $\begin{array}{c}37,588 \\
(26,912 \text { to } 48,390)\end{array}$ & $\begin{array}{c}241,227 \\
(-34,054 \text { to } 543,580)\end{array}$ \\
\hline $\begin{array}{l}\text { Global additional } \\
\text { deaths in } 2050\end{array}$ & $\begin{array}{c}84,697 \\
(-29,203 \text { to } 163,989)\end{array}$ & $\begin{array}{c}32,695 \\
(22,786 \text { to } 40,817)\end{array}$ & $\begin{array}{c}282 \\
\text { (195 to } 342)\end{array}$ & $\begin{array}{c}32,955 \\
(14,914 \text { to } 49,151)\end{array}$ & $\begin{array}{c}94,621 \\
(70,775 \text { to } 126,684)\end{array}$ & $\begin{array}{c}245,250 \\
(79,467 \text { to } 380,983)\end{array}$ \\
\hline
\end{tabular}

Note: the central estimate is the mean, based on three scenarios used with three different global climate models for a total of five runs. The uncertainty interval in brackets is the 
Table V.2: Proportion of the additional deaths provided by WHO (2014) assigned to each age-group

\begin{tabular}{cccccc}
\hline & Undernutrition & Malaria & Dengue & Diarrhoeal diseases & Heat waves \\
\hline $0-4$ & 1 & $1 / 14$ & $1 / 14$ & $1 / 3$ & 0 \\
$5-9$ & 0 & $1 / 14$ & $1 / 14$ & $1 / 3$ & 0 \\
$10-14$ & 0 & $1 / 14$ & $1 / 14$ & $1 / 3$ & 0 \\
$15-19$ & 0 & $1 / 14$ & $1 / 14$ & 0 & 0 \\
$\vdots$ & $\vdots$ & $\vdots$ & $\vdots$ & $\vdots$ & $\vdots$ \\
$60-64$ & 0 & $1 / 14$ & $1 / 14$ & 0 & 0 \\
$>65$ & 0 & $1 / 14$ & $1 / 14$ & 0 & 1 \\
\hline
\end{tabular}

Table V.3: Calibrated parameter value for all $\beta_{i}$ (scale $10^{-2}$ )

\begin{tabular}{ccccccc}
\hline$\beta_{1}$ & $\beta_{2}$ & $\beta_{3}$ & $\beta_{4}$ & $\beta_{5}$ & $\beta_{6}$ & $\beta_{7}$ \\
\hline 1.607 & 1.995 & 2.207 & 0.291 & 0.205 & 0.181 & 0.159 \\
& & & & & & \\
$\beta_{8}$ & $\beta_{9}$ & $\beta_{10}$ & $\beta_{11}$ & $\beta_{12}$ & $\beta_{13}$ & $\beta_{14}$ \\
\hline 0.142 & 0.118 & 0.092 & 0.066 & 0.049 & 0.035 & 0.143 \\
\hline
\end{tabular}

\section{Appendix VI - Sensitivity analysis of the excess mortality function as- sociated with climate change}

Table VI.1: Calibrated parameter value for all $\beta_{i}$ (scale $10^{-2}$ ) using Springmann et al.'s (2016) estimates for nutrition-related climate-induced deaths

\begin{tabular}{ccccccc}
\hline$\beta_{1}$ & $\beta_{2}$ & $\beta_{3}$ & $\beta_{4}$ & $\beta_{5}$ & $\beta_{6}$ & $\beta_{7}$ \\
\hline 6.46 & 1.995 & 2.200 & 0.292 & 0.205 & 0.184 & 0.159 \\
& & & & & & \\
$\beta_{8}$ & $\beta_{9}$ & $\beta_{10}$ & $\beta_{11}$ & $\beta_{12}$ & $\beta_{13}$ & $\beta_{14}$ \\
\hline 0.142 & 0.118 & 0.092 & 0.066 & 0.048 & 0.034 & 0.144 \\
\hline
\end{tabular}

\section{Acknowledgments}

This work benefited from the support of the Chair Energy and Prosperity, under the aegis of the Risk Foundation. We thank Emmanuel Bovari and Antoine Godin for their helpful comments on an earlier version of this article. We are also grateful to two anonymous referees for their fruitful comments and suggestions. Many thanks to Noah Ver Beek for 


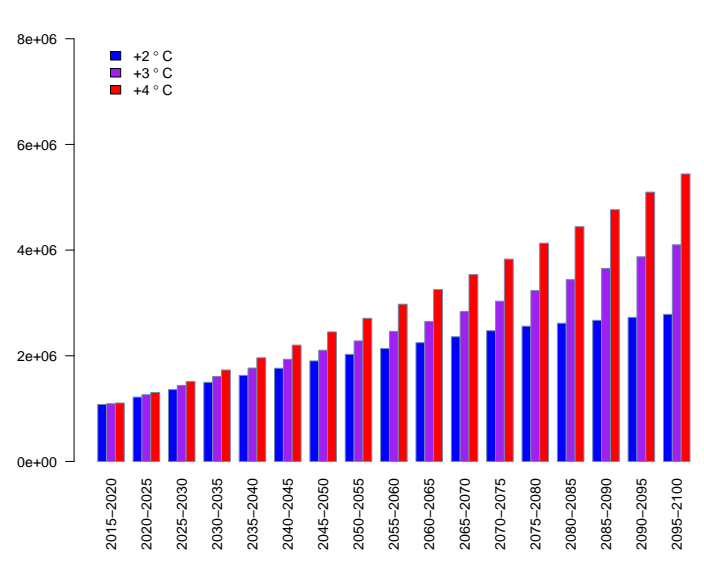

(a) $\theta=1$

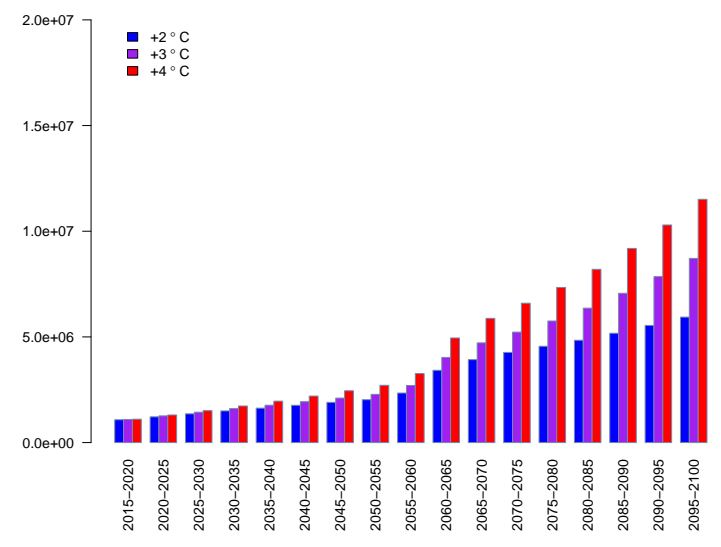

(c) $\theta=1$

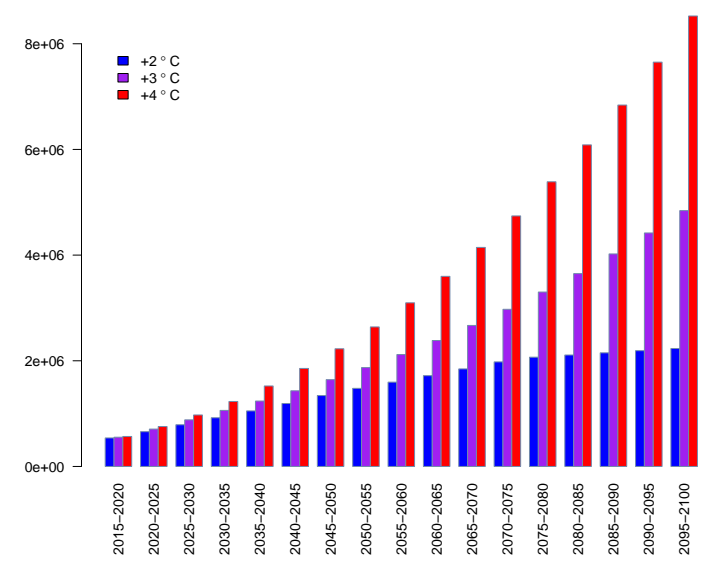

(b) $\theta=2$

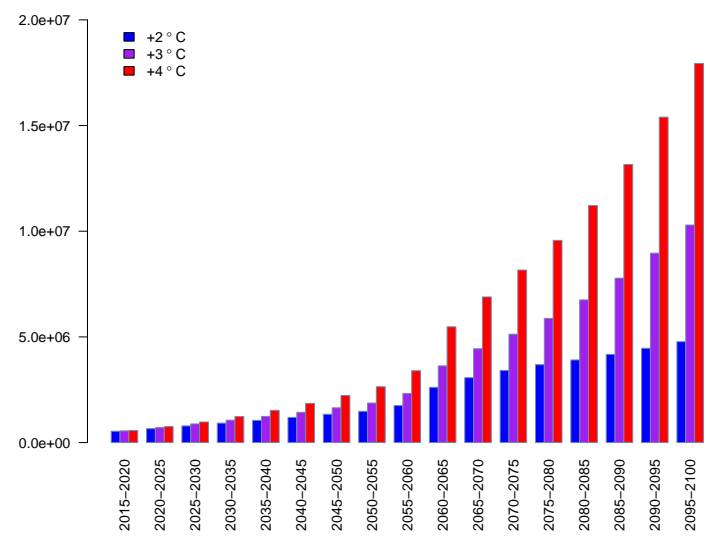

(d) $\theta=2$

Fig. VI.1. Climate change induced deaths every five years with exogenous (a-b) SSP2 and (c-d) GWP scenarios and Springmann et al.'s (2016) estimates for nutrition-related climate-induced deaths.

correcting the spelling and grammar of this text. All remaining errors and opinions are our own.

\section{Compliance with Ethical Standards}

The authors declare that they have no conflict of interest.

\section{References}

Akaev, A. A. and Sadovnichii, V. A. (2010). Mathematical model of population dynamics with the world population size stabilizing about a stationary level. Doklady Mathematics, 82(3):978-981.

Aral, M. M. (2014). Climate Change and Human Population Dynamics. Water Quality, Exposure and Health, 6(1-2):53-62. 
Becker, G. S. (1960). An Economic Analysis of Fertility. In Becker, G. S., editor, Demographic and Economic Change in Developed Countries. Princeton University Press, Princeton, NJ.

Becker, S. O., Cinnirella, F., and Woessmann, L. (2010). The trade-off between fertility and education: evidence from before the demographic transition. Journal of Economic Growth, 15:177-204.

Bolt, J., Inklaar, R., de Jong, H., and van Zanden, J. L. (2013). Maddison Project Database 2013. https://www.rug.nl/ggdc/historicaldevelopment/maddison/releases/maddison-projectdatabase-2013.

Cai, D. (2012). An economic growth model with endogenous carrying capacity and demographic transition. Mathematical and Computer Modelling, 55(3-4):432-441.

Carlson, E. and Tsvetarsky, S. (2000). Birthweight and infant mortality in Bulgaria's transition crisis. Paediatric and perinatal epidemiology, 14(2):159-162.

Carmichael, S., de Pleijt, A., van Zanden, J. L., and De Moor, T. (2016). The European Marriage Pattern and Its Measurement. Journal of Economic History, 76(1):196-204.

Chertow, M. R. (2000). The IPAT Equation and Its Variants. Journal of Industrial Ecology, 4(4):1329.

Cohen, J. E. (1995). Population Growth and Earth's Human Carrying Capacity. Science, 269:341346.

Cole, A. and Flenley, J. (2008). Modelling human population change on Easter Island far-fromequilibrium. Quaternary International, 184(1):150-165.

Cummins, N. (2013). Marital fertility and wealth during the fertility transition: rural France, 17501850. Economic History Review, 66(2):449-476.

de La Croix, D. and Michel, P. (2002). A Theory of Economic Growth: Dynamics and Policy in Overlapping Generations. Cambridge University Press, Cambridge, UK.

De Onis, M. (2000). Measuring nutritional status in relation to mortality. Bulletin of the World Health Organization, 78(10):1271-1274.

Di, Q., Wang, Y., Zanobetti, A., Wang, Y., Koutrakis, P., Choirat, C., Dominici, F., and Schwartz, J. (2017). Air pollution and mortality in the Medicare population. New England Journal of Medicine, 376(26):2513-2522.

Diaz, M. and Moore, f. (2018). Quantifying the economic risks of climate change. Nature Climate Change, 7(11):774-782.

Diebolt, C. and Perrin, F. (2013). From stagnation to sustained growth: the role of female empowerment. American Economic Review, 103(3):545-549.

Dolgonosov, B. M. (2016). Knowledge production and world population dynamics. Technological Forecasting and Social Change, 103:127-141.

Eberlein, R. L. and Thompson, J. P. (2013). Precise modeling of aging populations. System Dynamics Review, 29(2):87-101. 
Edenhofer, O., Pichs-Madruga, R., Sokona, Y., Farahani, E., Kadner, S., Seyboth, K., Adler, A., Baum, I., Brunner, S., Eickemeier, P., Kriemann, B., Savolainen, J., Schlömer, S., von Stechow, C., Zwickel, T., and Minx, J., editors (2014). Climate Change 2014: Mitigation of Climate Change. Contribution of Working Group III to the Fifth Assessment Report of the Intergovernmental Panel on Climate Change. Cambridge University Press, Cambridge, UK.

Ehrlich, P. R. (1968). The Population Bomb. Ballantine Books, New York, NY.

Franchini, M. and Mannucci, P. (2015). Impact on human health of climate changes. European Journal of Internal Medicine, 26(1):1-5.

Galor, O. and Mountford, A. (2008). Trading Population for Productivity: Theory and Evidence. Review of Economic Studies, 75(4):1143-1179.

Gasparrini, A. et al. (2017). Projections of temperature-related excess mortality under climate change scenarios. The Lancet Planetary Health, 1(9):e360-e367.

Goldewijk, K. K., Beusen, A., Doelman, J., and Stehfest, E. (2017). Anthropogenic land use estimates for the Holocene - HYDE 3.2. Earth System Science Data, 9:927-953.

Greenwood, J. and Seshadri, A. (2002). The U.S. Demographic Transition. American Economic Review, 92(2):153-159.

Guerrini, L. (2013). Analysis of an Economic Growth Model with Variable Carrying Capacity. International Journal of Mathematical Analysis, 7(26):1263-1270.

Guha-Sapir, D. and van Panhuis, W. (2003). The importance of conflict-related mortality in civilian populations. Lancet, 361(9375):2126-2128.

Harchaoui, S. and Chatzimpiros, P. (2018). Can agriculture balance its energy consumption and continue to produce food? A framework for assessing energy neutrality applied to French agriculture. Sustainability, 10(12):4624.

IPCC (2000). Special Report on Emissions Scenarios. A Special Report of Working Group III of the Intergovernmental Panel on Climate Change. Cambridge University Press, Cambridge, UK.

Jerven, M. (2012). An unlevel playing field: national income estimates and reciprocal comparison in global economic history. Journal of Global History, 7(1):107-128.

Kolstad, E. and Johansson, K. (2011). Uncertainties associated with quantifying climate change impacts on human health: a case study for diarrhea. Environmental health perspectives, 119(3):299305.

Kremer, M. (1993). Population Growth and Technological Change: One Million B.C. to 1990. Quarterly Journal of Economics, 108(3):681-716.

Lee, R. (2003). The Demographic Transition: Three Centuries of Fundamental Change. Journal of Economic Perspective, 17(4):167-190.

Luby, S. (2017). Clean water, clean hands or new vaccines? Journal of Infection, 74:S18-S22. 
Malthus, T. R. (1798). An Essay on the Principle of Population, as it Affects the Future Improvement of Society with Remarks on the Speculations of Mr. Godwin, M. Condorcet, and Other Writers. J. Johnson, London, UK.

Meadows, D. H., Meadows, D. L., Randers, J., and Behrens, W. (1972). The Limits to Growth: A Report for the Club of Rome's Project on the Predicament of Mankind. Universe Books, New York, NY.

Micó, J. C., Caselles, A., Soler, D., Sanz, M. T., and Martínez, E. (2008). A Side-by-Side Single Sex Age-Structured Human Population Dynamic Model: Exact Solution and Model Validation. Journal of Mathematical Sociology, 32(4):285-321.

Micó, J. C., Soler, D., and Caselles, A. (2006). Age-Structured Human Population Dynamics. Journal of Mathematical Sociology, 30(1):1-31.

Mora, C., Dousset, B., Caldwell, I., Powell, F., Geronimo, R., Bielecki, C., Counsell, C., Dietrich, B., Johnston, E., Louis, L., Lucas, M., McKenzie, M., Shea, A., Tseng, H., Giambelluca, T., Leon, L., Hawkins, E., and Trauernicht, C. (2017). Global risk of deadly heat. Nature Climate Change, 7:501-506.

Murphy, T. (2015). Old habits die hard (sometimes): Can département heterogeneity tell us something about the French fertility decline? Journal of Economic Growth, 20(2):177-222.

Navarro, A., Moreno, R., Jiménez-Alcázar, A., and Tapiador, F. J. (2017). Coupling population dynamics with earth system models: the POPEM model. Environmental Science and Pollution Research, pages 1-12.

Okuducu, M. B. and Aral, M. M. (2017). Knowledge based dynamic human population models. Technological Forecasting and Social Change, 122:1-11.

Peri-Rotem, N. (2016). Religion and Fertility in Western Europe: Trends Across Cohorts in Britain, France and the Netherlands. European Journal of Population, 32(2):231-265.

Pottier, A., Fleurbaey, M., Méjean, A., and Zuber, S. (2018). Integrated assessment of climate and population change: A first pass. Working Paper, pages 1-24.

Prados de la Escosura, L. (2016). Mismeasuring long-run growth: the bias from splicing national accounts—the case of Spain. Cliometrica, 10(3):251-275.

Ripple, W. J., Wolf, C., Newsome, T. M., Galetti, M., Alamgir, M., Crist, E., Mahmoud, M. I., and Laurance, W. F. (2017). World Scientists' Warning to Humanity: A Second Notice. BioScience, 67(12):1026-1028.

Sanz, M. T., Micó, J. C., Caselles, A., and Soler, D. (2014). A Stochastic Model for Population and Well-Being Dynamics. Journal of Mathematical Sociology, 38(2):75-94.

Shkolnikov, V., McKee, M., and Leon, D. (2001). Changes in life expectancy in Russia in the mid1990s. Lancet, 357(9260):917-921.

Smil, V. (2017). Energy and Civilization: A History. MIT Press, Cambridge, MA. 
Smith, M. and Myers, S. (2018). Impact of anthropogenic CO2 emissions on global human nutrition. Nature Climate Change, 8(9):834-839.

Sobotka, T., Skirbekk, V., and Philipov, D. (2011). Economic Recession and Fertility in the Developed World. Population and Development Review, 37(2):267-306.

Springmann, M., Mason-D’Croz, D., Robinson, S., Garnett, T., Godfray, H., Charles, J., Gollin, D., Rayner, M., Ballon, P., and Scarborough, P. (2016). Global and regional health effects of future food production under climate change: a modelling study. The Lancet, 387(10031):1937-1946.

Stocker, T., Qin, D., Plattner, G.-K., Tignor, M., Allen, S., Boschung, J., Nauels, A., Xia, Y., Bex, V., and Midgley, P., editors (2013). Climate Change 2013: The Physical Science Basis. Contribution of Working Group I to the Fifth Assessment Report of the Intergovernmental Panel on Climate Change. Cambridge University Press, Cambridge, UK.

Tapia Granados, J. and Diez Roux, A. (2009). Life and death during the Great Depression. Proceedings of the National Academy of Sciences of the United States of America, 106(41):17290-17295.

Tapia Granados, J. and Ionides, E. (2017). Population health and the economy: Mortality and the Great Recession in Europe. Health Economics, 26(12):e219-e235.

Tol, R. S. J. (2018). The Economic Impacts of Climate Change. Review of Environmental Economics and Policy, 12(1):4-25.

United Nations (2017a). World Population Prospects: The 2017 Revision, Methodology of the United Nations Population Estimates and Projections. Department of Economic and Social Affairs, Population Division.

United Nations (2017b). World Population Prospects: The 2017 Revision, Volume I: Comprehensive Tables. Department of Economic and Social Affairs, Population Division.

von Foerster, H., Mora, P. M., and Amiot, L. W. (1960). Doomsday: Friday, 13 November, A.D. 2026. At this date human population will approach infinity if it grows as it has grown in the last two millenia. Science, 132(3436):1291-1295.

WHO (2014). Quantitative risk assessment of the effects of climate change on selected causes of death, 2030s and 2050s. World Health Organization, Geneva, CH.

World Bank, T. (2018). World Bank Indicators, gross domestic product, constant 2010 US\$. https : //data.worldbank. org/indicator/NY. GDP.MKTP.KD. Accessed online 11-April-2018.

Wrigley, E. A. (2016). The Path to Sustained Growth: England's Transition from an Organic Economy to an Industrial Revolution. Cambridge University Press, Cambridge, UK. 